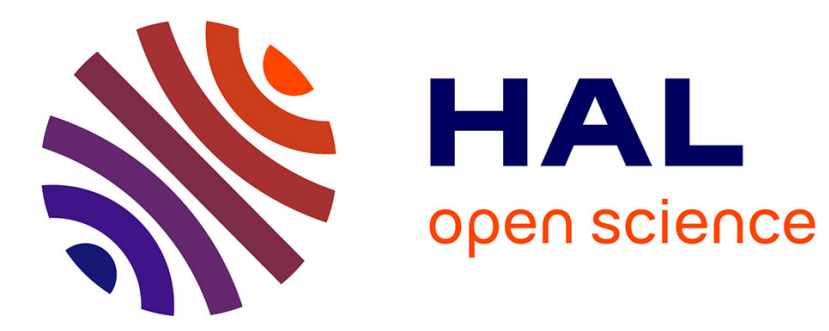

\title{
Experimental characterization of the initial zone of an annular jet with a very large diameter ratio.
}

\author{
Amélie Danlos, Gildas Lalizel, Béatrice Patte-Rouland
}

\section{To cite this version:}

Amélie Danlos, Gildas Lalizel, Béatrice Patte-Rouland. Experimental characterization of the initial zone of an annular jet with a very large diameter ratio.. Experiments in Fluids, 2013, pp.10.1007/s00348-012-1418-x. 10.1007/s00348-012-1418-x . hal-00767581

\section{HAL Id: hal-00767581 \\ https://hal.science/hal-00767581}

Submitted on 20 Dec 2012

HAL is a multi-disciplinary open access archive for the deposit and dissemination of scientific research documents, whether they are published or not. The documents may come from teaching and research institutions in France or abroad, or from public or private research centers.
L'archive ouverte pluridisciplinaire HAL, est destinée au dépôt et à la diffusion de documents scientifiques de niveau recherche, publiés ou non, émanant des établissements d'enseignement et de recherche français ou étrangers, des laboratoires publics ou privés. 


\title{
Experimental characterization of the initial zone of an annular jet with a very large diameter ratio.
}

\begin{abstract}
A.Danlos ${ }^{* *}$, G. Lalizel*, B. Patte-Rouland.
UMR 6614 CORIA, Complexe de recherche interprofessionnelle en aérothermochimie, Saint Etienne du Rouvray, France.

*Institut Pprime, Département Fluides, Thermiques et Combustion, axe COST, UPR 3346, ENSMA. Chasseneuil du Poitou, France.

** Laboratoire de Dynamique des Fluides (DynFluid), EA 92, Arts et Métiers ParisTech, Centre de Paris, Paris, France.

Abstract:

This article presents an experimental investigation of a large diameter ratio annular air jet by Particle Image Velocimetry (P.I.V.), Laser Doppler Anemometry (L.D.A.), hot wire anemometry and time-resolved tomography. Annular jets consist in a round nozzle with an obstacle placed in its center. These jets are thus defined by an external and an internal diameter corresponding to the round nozzle lips and the diameter of the obstacle respectively. The ratio between these two diameters defines the behavior of the flow across a characteristic diameter called diameter ratio. In most industrial applications these jets have large diameter ratios, superior to 0.7 .
\end{abstract}

Keyword: annular jet flow, wake, Kelvin-Helmholtz, instabilities, turbulence, Proper Orthogonal Decomposition.

\section{Introduction}

Turbulent jet flows are widely studied. As a matter of fact, they are commonly found in nature or in industrial processes. These flows have been frequently studied from an academic point of view because their geometries are simple and they can be considered as a source of momentum injected in a semi-infinite medium. Axisymmetric jet flows can have the shape of round or coaxial jets. If the fluid injection, in the central part of the coaxial jet is obstructed, the coaxial jet tends to an annular jet. The annular jet is thus characterized by the ratio $r$ between the outer diameter $D_{o}$ of the lips of the annulus and the inner diameter 
$D_{i}$ (see Figure 1 for details). This jet has initially the shape of an $e$ width annulus. Annular jet flows have successively the characteristics of a jet flow and of an axisymmetric wake flow. This induces a high sensitivity to instabilities. These two characteristics can also be used to increase or decrease the capacity of mixing for example. Annular jet flows can be observed in different stationary or non-stationary industrial flows. Many examples are presented: this geometry, called "bluff-body" allows a reduction of pollutant emission of $\mathrm{CO}$ and NOx and improves the stabilization of the flame. Sheffer et al. (1996) have proved that the optimal $r$ value used is between 0.5 and 0.92 and the order of magnitude of the fluid exit velocity $U_{0}$ is around $10 \mathrm{~m} / \mathrm{s}$. There are also some similarities between an annular jet flow and a flow around a valve during the admission cycle of an explosion engine. The $r$ value downstream from the valve is equal to about 0.88 in this case. The annular jet flow is also used in industrial processes for glass fibers generation. In the operating condition, we also could describe the use of annular jet for the cooling of workstation (Foss et al. 2003) or in non-pollutant system which allows a best elevation of the plume and an increase of dilution in chimney (Brendel 2002).

The annular jet flow characteristic parameters are the ratio $r$ between the inner diameter $D_{i}$ and the outer diameter $D_{o}$, the Reynolds number $\operatorname{Re}$, the half aperture angle $\alpha$ of the central obstacle and finally the shape of the exit velocity profile which should vary from a parabolic profile to a "top-hat" profile according to the exit momentum boundary layer thickness. The $\alpha$ angle can vary from $0^{\circ}$ for a cylinder to $90^{\circ}$ for a disc.

The main experimental studies focus on three aims: the determination of mean flow characteristics, the study of the transition from laminar to turbulent and the description of the instabilities of the flow. Chigier and Beer (1964) observed a toric shaped recirculation zone localized downstream the central obstacle. Davies and Beer (1971) have carried out a more precise study with measurements of both the mean flow and the fluctuating flow for various obstacle geometries and different values of $r$ ranging from 0.33 to 0.73 . They have shown that the length of the recirculation zone $L_{r z}$ decreases when the value of $r$ increases and that the turbulence level is very high downstream the obstacle. They also described the flow aerodynamics in three characteristic zones: a recirculation zone, a transition 
zone and a fully developed merging zone, where the evolution of the flow is similar to that of a round jet. Ko and Chan (1978) have used hot wire anemometry to study an annular jet flow with $r=0.45$ at a Reynolds number $\operatorname{Re}=57000$. They have observed that the mean velocity and turbulence intensity profiles are self-similar and they have measured that the length of the recirculation zone is equal to $L_{r z}=1.7 D_{o}$. They have also observed that the flow becomes fully developed at a shorter distance than a round jet ( 4 or $5 D_{o}$ instead of 8 ). Measurements for high diameter ratio $r$ do not seem to have been carried out in the recirculation zone. Aly and Rashed (1991) have been interested in studying the fully developed merging zone for $r=0.91$. They compared the mean velocity and the turbulent profile with a round jet flow.

The flow transition, from laminar to turbulent, have been studied by $\mathrm{Li}$ and Tankin (1987). They have described the effect of the Reynolds number and the effect of the diameter ratio $r$, varying from 0.32 to 0.72 , on the flow topology. They have observed that the length $L_{r z}$ of the recirculation zone initially depends on the Reynolds number and then not.

The description of instabilities has been studied by Ko and Leung (1982) and Ko et al. (1988). They observed three types of instabilities. The first one is located in the external mixing layer induced by the shear between the annular potential core flow and the surrounding air. The second instability occurs in the internal mixing layer between the annular potential core and the recirculation zone. The last instability takes place in the wake of the obstacle. Ko et al. (1988) observed a strong interaction between structures in the internal mixing layer and the wake layer.

According to these experimental studies, it is shown that the annular jet flow can be divided in three different zones: an initial merging zone characterized by the annular potential core and the recirculation zone, a transition zone and a fully developed merging zone where the flow has turbulent jet flow characteristics. It appears that experimental studied annular jet flows have a diameter ratio $r$ smaller than 0.7. With this geometry, annular jet flows are quite stable and the recirculation zone is confined inside the annular potential core. Indeed, despite the presence of instabilities in mixing layers and in the wake of the central obstacle, the recirculation zone contributes to stabilize the flow on the whole. This is advantageous, especially for burners, where this geometry represents a flame 
stabilization device. Most of studies on the annular jet present a two-dimensional analysis of the annular jet flow. However, some studies investigate threedimensional instabilities of the flow and highlight an asymmetry of the flow (Del Taglia (2003), Vanierschot et al. (2011)). There are no precise known experimental description of the recirculation zone flow for a diameter ratio higher than 0.7 . However, mainly industrial processes use a very large annular jet flow diameter ratio. In this article, an experimental study of annular jet flows with $r>0.7$ is performed. Using P.I.V. and L.D.A. measurements makes it possible to fully characterize these particular flows.

\section{Experimental setup.}

The annular jet facility is reported on figure 1. The nozzle is geometrically characterized by the outer diameter $D_{o}$ equal to $53.88 \mathrm{~mm}$ and the inner diameter $D_{i}$ equal to $48.75 \mathrm{~mm}$. The width of the nozzle output $e$ is thus equal to 2.565 $\mathrm{mm}$ and the diameter ratio $r$ is equal to 0.905 . The choice of the curvature of the external and internal parts of the nozzle has been determined from Hussain's work (1976). The contraction ratio, defined as the ratio between the inlet section and the outlet section of the nozzle $\left(\mathrm{D}_{\mathrm{b}}{ }^{2} / \mathrm{D}_{\mathrm{o}}^{2}\right)$, is equal to 102 . The diameter $\mathrm{D}_{\mathrm{b}}$ used to characterize the contraction ratio is defined at a distance $L=120 \mathrm{~mm}$ from the nozzle outlet. The flow is fed with the compressed air laboratory facility. The air is clean and dry. The flow is regulated with a sonic neck. Different facilities are used to reduce turbulence intensity of the annular potential core: a fluidized bed of glass balls, five layers of silk filters and finally a honeycomb. All these elements allow the homogenization and the reduction of the turbulence level of the flow at the nozzle exit. 


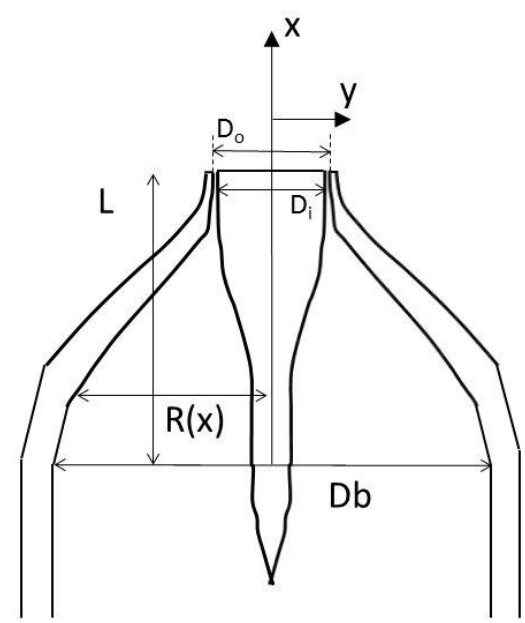

(a)

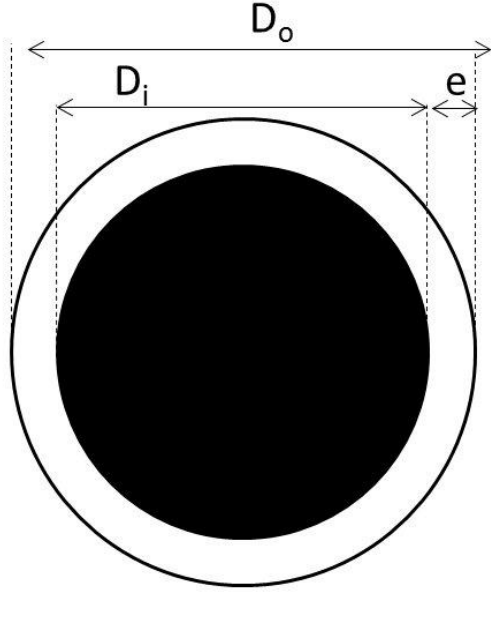

(b)

Fig.1 Annular jet facility. (a) Front view, (b) Top view

The non-dimensional longitudinal direction called $x *$ is defined by $x^{*}=\frac{x}{D_{o}}$ and the transversal coordinate called $y^{*}$ is defined by $y^{*}=\frac{y}{D_{o}} \cdot U$ is the mean longitudinal velocity and $U_{0}$ is the exit longitudinal velocity. $U^{*}$ is the nondimensional longitudinal velocity, determined by the ratio between $U$ and $U_{0} . V$ and $V^{*}$ are respectively the mean transversal velocity and the non-dimensional transversal velocity, determined by the ratio between $V$ and $U_{0}$. Root-meansquare values of $U$ and $V$ are $u^{\prime}$ and $v^{\prime}$. It has been proved by Godard et al. (1998) that the flow is fully symmetric in the exit section.

In this study, three exit Reynolds numbers $\mathrm{Re}_{e}$ have been investigated: 1368, 2565 and 5130, which corresponds to exit velocities equal to 8, 15 and $30 \mathrm{~m}^{-1} \mathrm{~s}^{-1}$. The exit longitudinal velocity profile has been measured with hot-wires anemometry at a distance from the exit evaluated to $50 \mu \mathrm{m}$. The results show that the turbulence intensity in the potential core is equal to $0.7 \%$ and that the ratio between the outer diameter $\mathrm{D}_{\mathrm{o}}$ and the momentum boundary layer $\theta_{e}$ ranges from 360 to 430.

$$
\theta_{e}=\int_{0}^{\infty} \frac{U(y)}{U_{0}}\left(1-\frac{U(y)}{U_{0}}\right) d y
$$


All these results prove that the initial velocity profiles of the flow have a « top hat » shape. The turbulent spectra do not show any characteristic peaks due to the acoustic resonance of the jet facility.

Different techniques, intrusive and non-intrusive, have been used to study the flow. Temporal visualizations of the flow have been performed in the recirculation zone until $x^{*}=1.7$ using a 4540 Kodak camera. The flow was seeded by $1 \mu \mathrm{m}$ olive oil droplets. The oil was illuminated by a Ar/Ion 5 watts continuous wave laser, 400 images of the flow were acquired with a resolution of $256 \times 256$ square pixels at a frequency of $4500 \mathrm{~Hz}$ and from two points of view: in the axisymmetric longitudinal plane and in different transversal planes at distances from the exit $x^{*}$ equal to $0.06,0.26,0.41,0.52,0.65$ and 0.93 .

A double pulsed Nd-Yag laser is used to set up the light sheet of the P.I.V. setup. The output energy is nearly $30 \mathrm{~mJ}$ for each laser pulse. The wavelength is $532 \mathrm{~nm}$. The laser beams are focused onto a sheet across the median plane of the annular jet by one cylindrical ( $f=-0.02 m)$ and one spherical $(f=0.5 m)$ lens. The time delay between two pulses, which depends on the exit velocity $U_{0}$ and the size of the observation fields, varies from 2 to $20 \mu \mathrm{s}$. Two different observation fields have been studied. The first one, the plane $P_{1}$, is $60 \times 54 \mathrm{~mm}^{2}$ and is centered on the recirculation zone. The second observation field, the plane $P_{2}$, is $28 \times 25 \mathrm{~mm}^{2}$ to resolve the two mixing layers of the annular jet. The video images are recorded with a LAVISION Flow Master 3S camera. The frame grabber digitizes each field in $1280 \times 1024$ pixels with 12 bits gray levels. The acquisition frequency is $4 \mathrm{~Hz}$. Interrogation of the recorded images is performed by two-dimensional digital cross correlation analysis using "Davis 6.2.2.". For velocity fields of $P_{1}$ plane, the sampling window is $32 \times 32$ pixels $\left(1.72 \times 1.72 \mathrm{~mm}^{2}\right)$ with a $60 \%$ overlap with the next window. In $P_{2}$ plane, the window is 16 by 16 pixels $(0.377 \times 0.377 \mathrm{~mm})$ with $50 \%$ overlap. The flow and the surrounding air are seeded with olive oil droplets. A study of the droplet size distribution has shown that the mean diameter is equal to $1.5 \mu \mathrm{m}$. For each exit velocity, 500 P.I.V. images have been recorded. We observed less than $1 \%$ of false vectors calculated in the flow. We used these 500 velocity fields to calculate the different parameters such as mean velocity, velocity variance, or vorticity fields. Computation of the convergence of the two first moments of the velocity shows that 500 velocity fields are enough for 
minimizing the relative error. This error on the first order moment is within $4.5 \%$ and the relative error on the second order moment is about $2 \%$ (figure 2 ).

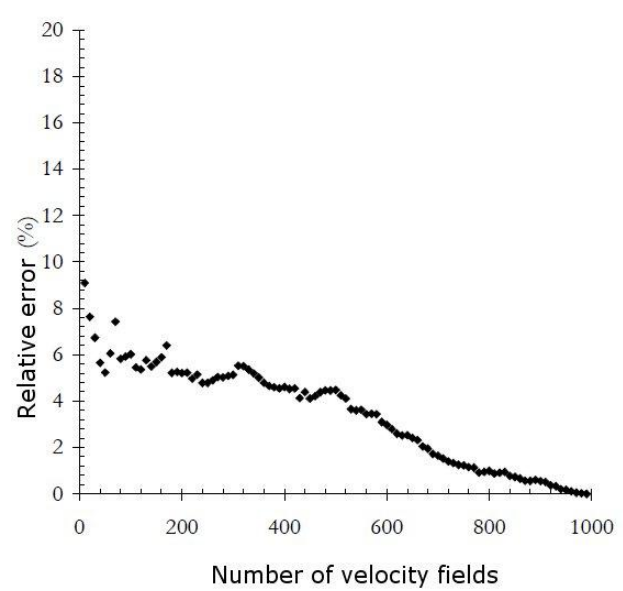

a)

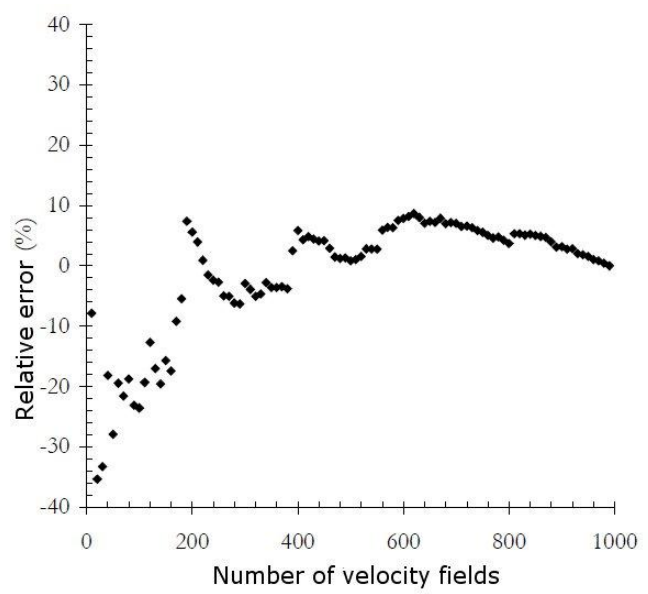

b)

Fig.2 Statistical relative error on the (a) first and (b) second order moments, according to the number of velocity fields, with $\mathrm{U}_{0}=8 \mathrm{~m} \cdot \mathrm{s}^{-1}$

The Laser Doppler Anemometry (L.D.A.) experimental setup is composed of a 5 W Ion/Ar continuous wave laser and a two components TSI system. The longitudinal velocity $U$ is obtained from the green beam $(514.5 \mathrm{~nm})$ and the transversal velocity $V$ from the blue $(488 \mathrm{~nm})$. The diameter of the acquisition volume is equal to $82 \mu \mathrm{m}$ for the green beams and $78 \mu \mathrm{m}$ for the blue ones. The lengths of the volume are respectively $1.19 \mathrm{~mm}$ and $1.13 \mathrm{~mm}$. Acquisitions of the two components are not time-correlated. The total number of acquired velocities is equal to 7680 . The mean frequency is always up to 3000 particles per second. The validation rate of particles counting is up to $96 \%$. Considering the results obtained by 2D L.D.A., we have decided to complete the study with 3D L.D.A measurements on slices of annular jet in the recirculation zone. These measurements use an Innova COHERENT Laser with a TSI Colorburst Model 9201 to divide the laser beam in three pairs of beams with different wave lengths in order to create a $0.13 \times 0.13 \times 1.8 \mathrm{~mm}^{3}$ measurement volume. This allows to obtain time-correlated values. The volume is moved on 8 profiles of 33 measurement spots and each measure is validate by 50000 particles with a validation rate superior to $85 \%$.

The experimental setup of hot-wire anemometry is composed of a single wire probe, a TSI IFA 300 processor and an acquisition PC. The diameter of the wire, made of $10 \% \mathrm{Pt}-\mathrm{Rh}$ is $2.5 \mu \mathrm{m}$. The calibration of the wire is realized with a 
laminar $8 \mathrm{~mm}$ round jet blower. The experimental calibration is modeled with a cubic spline interpolation function. An external temperature correction is applied during the acquisition. The acquisition frequency is $20 \mathrm{kHz}$ and the duration of acquisition samples is equal to $13 \mathrm{~s}$.

\section{Proper Orthogonal Decomposition (P.O.D.) analysis used as an efficient post-processing of P.I.V. measurements}

P.O.D. analysis is used for the study of many signals but this technique represents a powerful tool to highlight coherent structures and to observe their features and their evolution in a flow by applying this technique on two-dimensional velocity fields (Holmes1996). These sets of data generate therefore an optimal set of basis functions in modal base. In this context P.O.D. analysis uses physical functions with finite kinetic energy equivalent to the square integrable function. As coherent structures should be the structures that have the largest mean square projection, the method implies to find the functions $\Phi$ the most "similar" to the set of velocity fields $U$. It boils down to find the function that maximize the averaged projection of $U$ onto $\Phi$, normalized as:

$$
\underset{\Phi \in L^{2}([0,1])}{\operatorname{ax}} \frac{\left\langle(U . \Phi)^{2}\right\rangle}{\|\Phi\|^{2}}
$$

Where \langle\rangle represents an ensemble average, and $\|$.$\| denotes the L^{2}$-norm: $\|\Phi\|=(\Phi, \Phi)^{1 / 2}$. After developing calculations, we obtain the following eigenvalue problem :

$$
\iint_{D}\left\langle U(x) U^{*}\left(x^{\prime}\right)\right\rangle \Phi\left(x^{\prime}\right) d x^{\prime}=\lambda \cdot \Phi(x)
$$

Where $\lambda$ is the eigenvalues set, and $U^{*}$ represents the complex conjugate of $U$, with $x, x^{\prime} \in D$. It could also be written in another way:

$$
\iint_{D} R_{i j}\left(x, x^{\prime}\right) \cdot \Phi_{j}^{(n)}\left(x^{\prime}\right) d x^{\prime}=\lambda^{(n)} \Phi_{i}^{(n)}(x)
$$


$D$ defines the two-dimensional domain of the velocity fields and $R$ is the averaged two-point correlation tensor defined by :

$$
R_{i j}\left(x, x^{\prime}\right)=\left\langle U_{i}(x) \cdot U_{j}\left(x^{\prime}\right)\right\rangle
$$

As the eigenfunctions $\Phi$ build a complete base, each velocity field $U_{i}$ could be written as:

$$
U_{i}=\sum_{k=0}^{N-1} a_{k, i} \Phi_{k}
$$

The form of the eigenfunctions is therefore :

$$
\Phi=\sum_{k=0}^{M-1} a_{k} U^{(k)}
$$

With the decomposition of each velocity field as a linear combination of proper modes $\phi$, we can calculate the eigenvalue $\lambda_{\mathrm{k}}$ corresponding to the energy contained in the mode $\mathrm{k}$. These eigenvalues are ordered by $\lambda_{\mathrm{i}}>\lambda_{\mathrm{i}+1}$. In other words, each eigenfunction corresponds to a proper mode skeleton which has a weight in the flow described by the eigenvalue.

$$
u_{i}(x, y)=\sum_{k=0}^{N-1} a_{i, k} \Phi_{k}(x, y) \text { and } \delta_{k k^{\prime}} \lambda_{k}=\left\langle a_{k} a_{k^{*}}{ }^{*}\right\rangle
$$

Where $a_{i, k}$ is the reconstruction coefficient.

\section{Experimental results.}

\section{Study of the initial zone.}

This study focuses the complete recirculation zone characterization. The evolution of $U_{m} / U_{0}$ is studied for $U_{0}$ equal to 8,15 and $30 \mathrm{~m}_{\mathrm{s}} \mathrm{s}^{-1}$ in figure 3 , where $U_{m}$ is the maximum longitudinal velocity of a transverse velocity profile. 


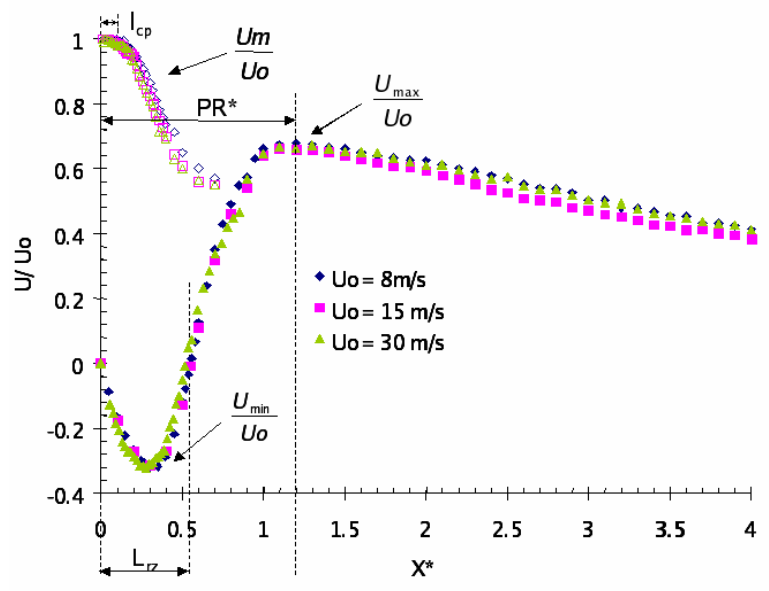

Fig.3 Evolution of the dimensionless maximum longitudinal velocity of a transverse velocity profile $U_{m} / U_{0}$ and evolution of the longitudinal variation of longitudinal velocity on the central axis of the jet ( $\mathrm{PR} *$ is the non-dimensional longitudinal position of the attachment point)

The evolution of the maximum longitudinal velocity of a transverse profile $U_{m}$ according to $x^{*}$ is plotted on figure 3. The evolution is similar, whatever $U_{0}$. From this graph, the length of the annular potential core is $l_{c p}=0.16 D_{o}$ or $l_{c p}=3.4 e$, where $e$ is the thickness of the ring. $l_{c p}$ is defined as the distance $x *$ where $U_{m}=0.97 U_{0}$. We can observe the presence of an axisymmetric recirculation zone characterized by negative longitudinal velocities near the obstacle. Its length, $L_{r z}$ denotes the longitudinal localization of the stagnation point with null velocity. For an annular jet with a 0.91 diameter ratio, the length of the recirculation zone is thus greater than the length of the annular potential core, contrary to previous annular jet study (Ko 1978, Chigier 1964). For annular jets with a diameter ratio $0.56 \leq r \leq 0.85$, Li et al. (1987) study the transition from laminar to turbulent of these flows. From their measurements, this transition was estimated to appear when $700<\operatorname{Re}_{e}<800$. Huang et al. (1994 present a study about the influence of the Reynolds number on the annular jet flow with a diameter ratio $r=0.66$, with visualizations in a longitudinal plane of the jet. The "closed toroid type" flow appears for $\operatorname{Re}_{D_{i}}>455$. This is the established flow mode of the annular jet. The separation free surface is closed and merges with the jet axis to form a recirculation bubble with a toroidal shape. Thus, if the Reynolds number is higher than 800 , the recirculation zone length depends mostly on the diameter ratio 
$r=D_{i} / D_{o}$. The Reynolds number influence is only considered in the second order.

Downstream the end of the annular jet potential core, $U_{m}$ decreases linearly in the studied zone. The decrease rate, as a function of $e$, is equal to -0.0564 . This value is lower than the value for a round jet, -0.0654 (Crow 1971) and higher than that of a plane jet, -0.0411 (Weiss 1999). $L_{r z}$ decreases with $U_{0}$. The ratio $L_{r z} / D_{i}$ is equal to $0.611,0.606$ and 0.578 for respectively $U_{0}$ equal to 8,15 and $30 \mathrm{~m}^{-1}{ }^{-1}$. Two forces exist in recirculation zone: inertial forces $F_{i}$ and pressure forces $F_{p}$. Inertial forces tend fluid to get further the obstacle, contrary to pressure forces who keep fluid near the obstacle. These forces $F_{i}$ and $F_{p}$ can be defined by:

$$
F_{i} \approx \rho\left(D_{o}^{2}-D_{i}^{2}\right) U_{0}^{2} \text { and } F_{p} \approx\left(P_{a t m}-P_{r z}\right) D_{i}^{2}=\Delta P \cdot D_{i}^{2}
$$

Where $P_{r z}$ is the maximal depression localized at center of the recirculation zone and $P_{a t m}$ is atmospheric pressure. So, when exit velocity increases, inertial forces increase and recirculation zone length is larger. Experimental values of $L_{r z}$, plotted in figure 4, are in good agreement with those available in Del Taglia bibliographic review (2003). It shows that $L_{r z}$ decreases linearly as a function of blockage ratio $r^{2}=\frac{D_{i}^{2}}{D_{o}^{2}}$. The ratio between inertial forces and pressure forces can be derived as:

$$
\frac{F_{i}}{F_{p}} \approx \frac{\left(D_{o}^{2}-D_{i}^{2}\right)}{D_{i}^{2}} \frac{U_{0}^{2}}{\Delta P} \approx\left(\frac{1}{r^{2}}-1\right) \frac{U_{0}^{2}}{\Delta P}
$$

Where $\Delta P$ denotes the pressure gradient. Behind a disk, the variation of $\frac{U_{0}^{2}}{\Delta P}$ as a function of $\theta$ angle, defined between measurement localization and main axe of flow, does not vary with the Reynolds number. In this case, ratio between $F_{i}$ and $F_{p}$ only depends on inverse of $r^{2}$. So when $r$ increases, $L_{r z}$ decreases. This is consistent with experimental results compiled by Del Taglia (2003). 


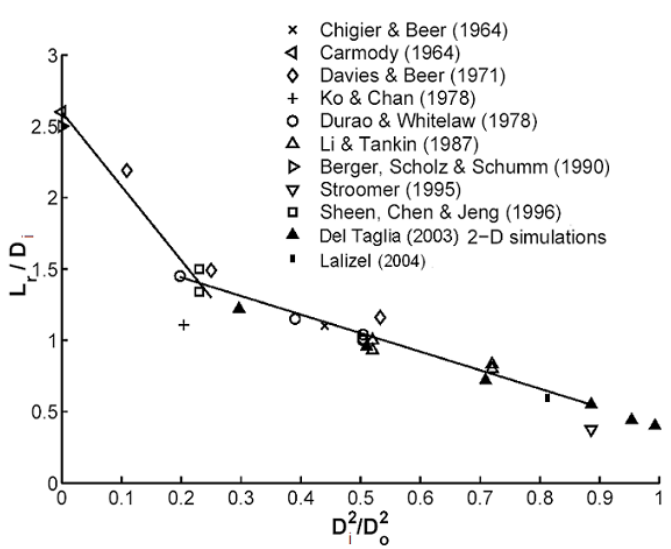

(a)

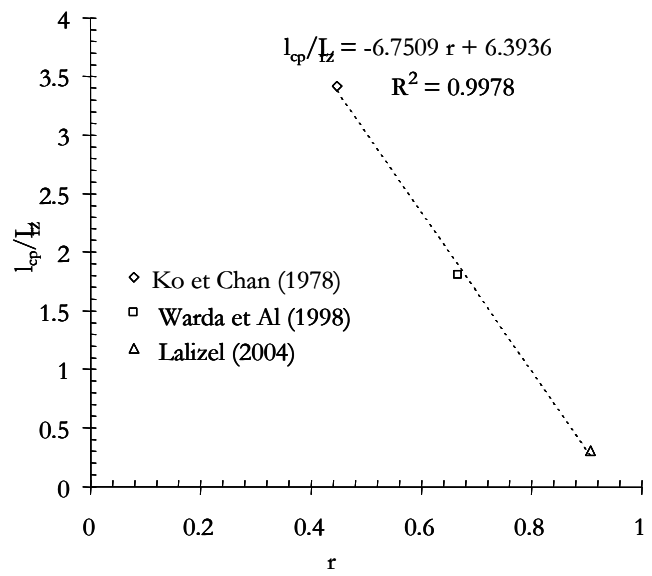

(b)

Fig.4 (a) Bibliographic review of recirculation zone length according to blockage ratio $D_{i}^{2} / D_{o}^{2}$, from Del Taglia (2003). (b) Variations of $l_{c p} / L_{r z}$ as a function of $r$ diameter ratio

The variation of ratio between $l_{c p}$ and $L_{r z}$ as a function of $r$ is plotted on figure 4 (b) from Ko and Chan (1978) and Warda et al. (1998). $l_{c p} / L_{r z}$ is linear and can be described by the relation:

$$
\frac{l_{c p}}{L_{r z}}=-6.8 r+6.4
$$

Patte-Rouland et al. (2001), Danlos et al. (2008) have shown that rms transverse velocity is greater around the stagnation point localization (figure 5) and the ratio between transverse and longitudinal velocity rms shows that longitudinal rms can be neglected in comparison to transverse one. This figure 5 seems to highlight a slight asymmetry of the flow which will be discussed in the last part of this study.

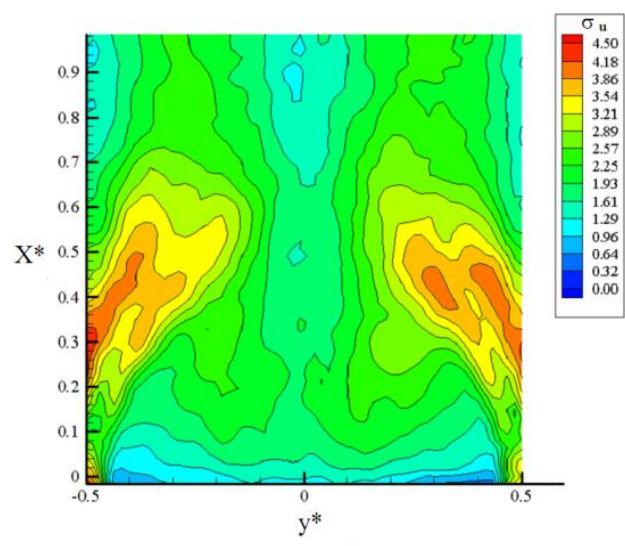

(a)

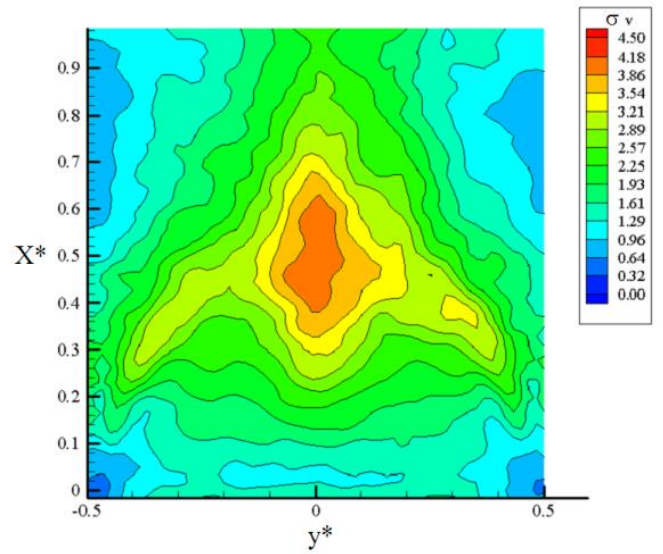

(b) 
Fig.5 rms of (a) longitudinal velocity and (b) transverse velocity of the initial zone of annular jet for $U_{0}=30 \mathrm{~m} / \mathrm{s}$.

The stagnation point is submitted to large transverse fluctuations. From the stagnation point, the longitudinal evolution of the axial ratio between rms value $v^{\prime}$ and rms value $u^{\prime}$ are greater than 1 . This zone is mainly characterized with very large transverse velocity fluctuations. An application of Proper Orthogonal Decomposition (P.O.D.) on a statistic of instantaneous velocity fields have already shown that the stagnation point localization is spatially correlated with an instantaneous asymmetry of the recirculation zone (Patte-Rouland 2001, Danlos 2008). This phenomenon has been correlated to the topology of the P.O.D. mode number 1 which showed a rotation of fluid behind the internal $D_{i}$ disc and a transverse flow around the mean stagnation point.

The time between two P.I.V. velocity fields measurement is too long to resolve the periodic evolution of the asymmetry of the flow. Temporal measurements with L.D.A have been conducted around the mean stagnation point. Gaster and Bardbury (1976) have shown that the choice of the transverse velocity is the most interesting to resolve instabilities behind an obstacle: the amplitude of the peak of frequency spectra of transverse velocity is 10 times more intense than the longitudinal one. From the temporal evolution of $V$, we have computed the autocorrelation coefficient $\rho$ for the three exit velocities from "slotting" technique (Van Maanen 1999). $\rho$ is defined as:

$$
\rho(\tau)=\frac{R(\tau)}{\overline{u^{\prime 2}}} \text { where } R(\tau)=\int_{0}^{\infty} u^{\prime}(t) u^{\prime}(t+\tau) d t
$$

The evolution of $\rho$ for $U_{0}=8 m \cdot s^{-1}$ is plotted on figure 6(a) and (b). The mean acquisition frequency $f$ is $9982 \mathrm{~Hz}$. The total decrease of $\rho$ on figure 6(a) shows two different zones for $\tau<75 \mathrm{~ms}$. The first one, for $\tau<10 \mathrm{~ms}$, is characteristic of the turbulence of the flow at the mean stagnation point: $\rho$ decreases very fast. For $\tau>10 \mathrm{~ms}, \rho$ decreases slower which is characteristic of the presence of coherent structures. The complete evolution of $\rho$ is represented on figure 6(b). $\rho$ is similar to an autocorrelation function of a periodic evolution as behind a cylinder in a Benard-Von Karman street for example. An application of a Discrete Fourier 
Transform shows a frequency peak at $f_{s p}=39 \mathrm{~Hz}$. The value of $f_{s p}$ for $U_{0}$ equal to 15 and $30 \mathrm{~m} \cdot \mathrm{s}^{-1}$ are respectively $f_{s p}=72 \mathrm{~Hz}$ and $f_{s p}=150 \mathrm{~Hz}$.

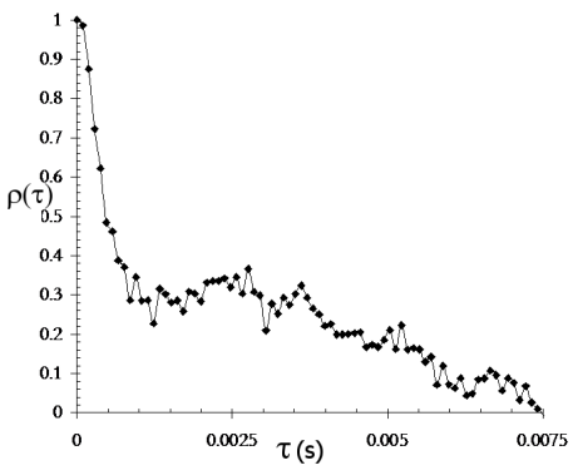

a)

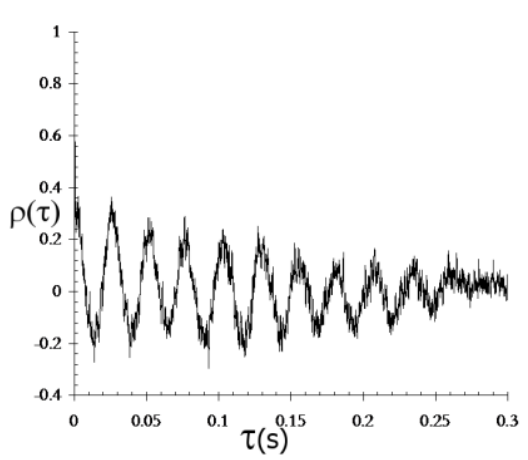

b)

Fig.6 Evolution of $\rho(\tau)$ for $U_{0}=8 \mathrm{~m} / \mathrm{s}$ on stagnation point, $f=9982 \mathrm{~Hz}$ and $T=0.73 \mathrm{~s}$

The Strouhal number $S t_{D_{i}}=\frac{f_{s p} D_{i}}{U_{0}}$, based on the exit velocity $U_{0}$, on frequency $f_{s p}$ and the length of the obstacle $D_{i}$, is constant and equal to 0.23 . This is in accordance with the Strouhal number of the wake behind the inner disc $D_{i}$. The large rms transverse velocity of the mean stagnation point is thus the consequence of a non stationnary Lagrangian displacement of the stagnation point due to instabilities of the recirculation zone.

\section{Recirculation zone mixing layer study.}

A mixing layer consists in a shear layer submitted to a strong shear that creates vortices spring from a distortion of the interface between two parallel flows with different velocities. When these two flows have a same viscosity and a same density, Kelvin-Helmholtz is the most probable instability developed in the shear layer. Vortices linked to this instability become larger downstream the flow and promote mixing between fluids by increasing shear layer thickness (Brown and Roshko 1974). If the initial balance of the flow is disturbed, a tiny velocity fluctuation bends out the interface between the two fluid streams. This interface is then submitted to an oscillation. As Bernoulli equation predicts, pressure is different on each side of the interface. This difference amplifies the oscillation. However, the interface cannot grow endlessly. The instability saturates and the interface wraps itself in order to generate a Kelvin-Helmholtz vortex. In a vortex 
wake, an induction area can then increase the velocity of the upstream vortex until these two vortices pairing. This combination creates a vortex with a larger size (about twice as large as parents vortices). This pairing phenomenon plays a role in the enlargement of the mixing layer. Kelvin-Helmholtz vortices are primary structures developed from primary instability. By using a second order threedimensional non-linear analysis of mixing layer instabilities, Benney (1961) highlights a longitudinal vorticity. These instabilities are called three-dimensional instabilities or secondary instabilities.

Bernal and Roshko (1986) identified these three-dimensional structures that are created in the braid, between Kelvin-Helmholtz vortices. Phenomena which lead the shear layer to develop a three-dimensional structure can be a tiny deflection of transversal vorticity lines caused by a disturbance upstream the flow (like ruggedness on the injector surface), primary structures pairing (radial velocity varies with pairing phenomena which stretch and tense Kelvin-Helmholtz vortices, then longitudinal vorticity is created), or other instabilities linked to primary structures itself like translative instability suggested by Pierrehumbert and Widnall (1982). The small disturbance is then amplified by the slick stretching caused by primary vortices. Secondary vorticity interacts then with primary vorticity of Kelvin-Helmholtz vortices that loose their shape and secondary vorticity line rolls up around them and creates a three-dimensional hairpin like structure. These ones collapse in order to create contrarotative vortices pairs. Secondary structures may reach higher energy levels than primary structures. These results for a plane-mixing layer can be extended to an annular jet flow.

The rms longitudinal velocity field shows that the maximum velocity fluctuations are localized around the annular exit of the jet and these values are nearly $15 \%$ of output velocities (figure 7). There are two mixing layers: the external one, between the annular potential core of the flow and the stagnant surrounding air, and the internal one, between the potential core and the recirculation zone. The mean velocity field of the mixing layer is plotted on figure 7(a). Some streamlines of the flow are also plotted. W can observe that the flow converges through the central axis of the jet from a distance $x^{*}=0.15$. 


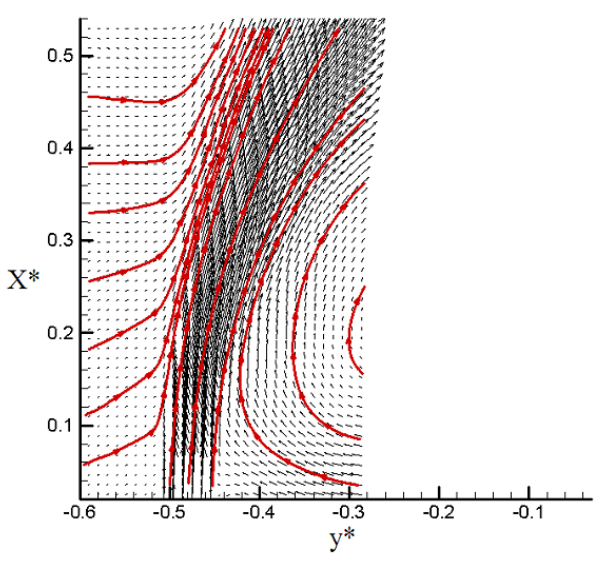

a)

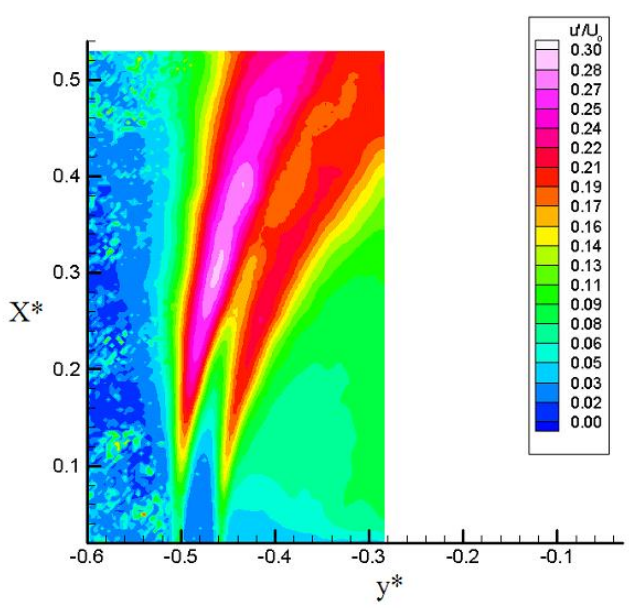

c)

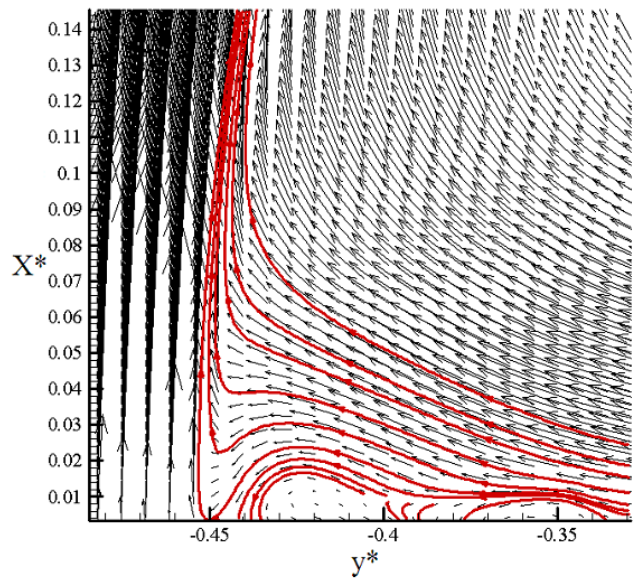

b)

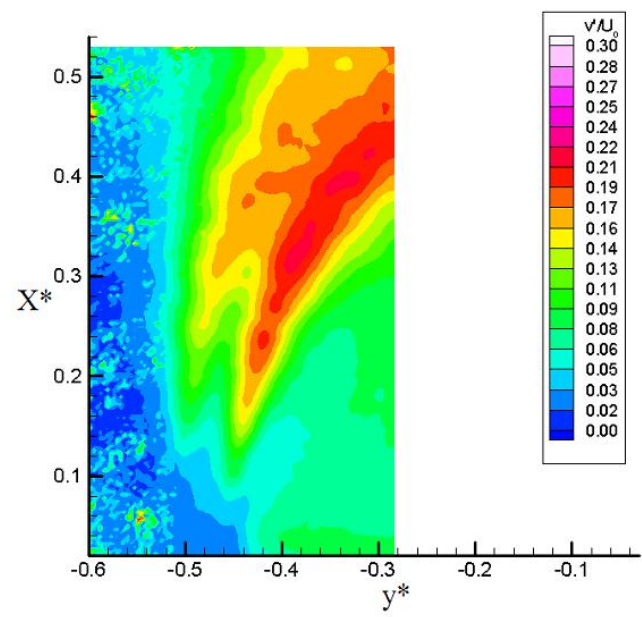

d)

Fig.7 a) Mean velocity field, b) close-up of the mean velocity field, c) field of longitudinal velocity rms, d) field of transverse velocity rms for $U_{0}=8 \mathrm{~m} . \mathrm{s}^{-1}$

Vorticity $W$, strain stress $\sigma_{s}$, shear stress $\sigma_{n}$ and Weiss coefficient $Q$ have been also computed from instantaneous P.I.V fields. $Q$ is proportional to pressure Laplacian and defined by :

$$
Q=\omega^{2}-S^{2} \text { and } S^{2}=\sigma_{s}^{2}+\sigma_{n}^{2}
$$

Where $\sigma_{s}$ and $\sigma_{n}$, are defined by :

$$
\sigma_{n}=\frac{\partial U}{\partial x}-\frac{\partial V}{\partial y} \text { and } \sigma_{s}=\frac{\partial V}{\partial x}+\frac{\partial U}{\partial y}
$$


Mean fields of vorticity, strain stress and shear stress are plotted on figure 8 for $U_{0}$ equal to $8 m \cdot s^{-1}$.

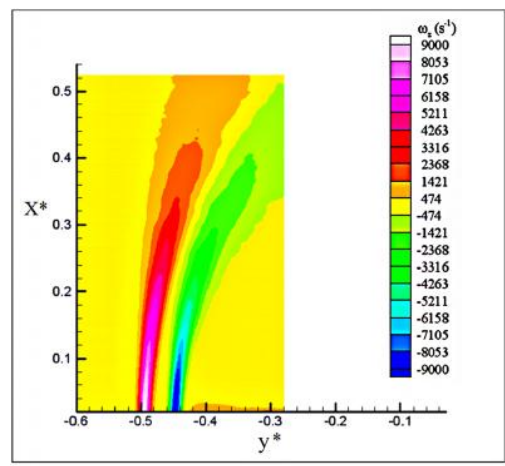

a)

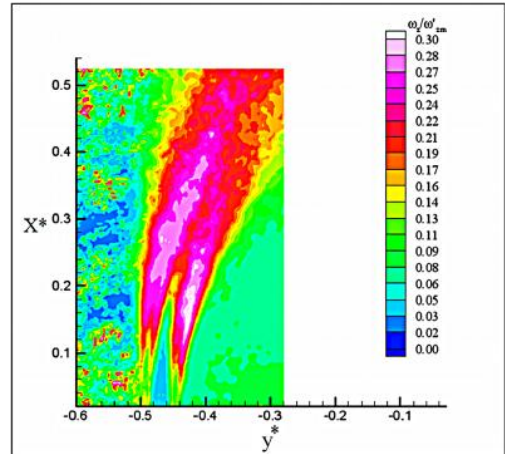

$\left.\mathrm{a}^{\prime}\right)$

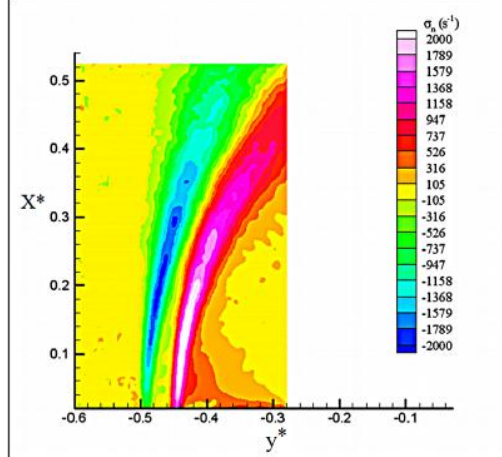

b)

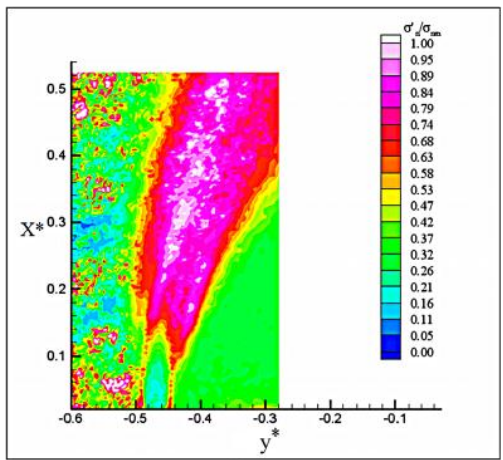

$\left.b^{\prime}\right)$

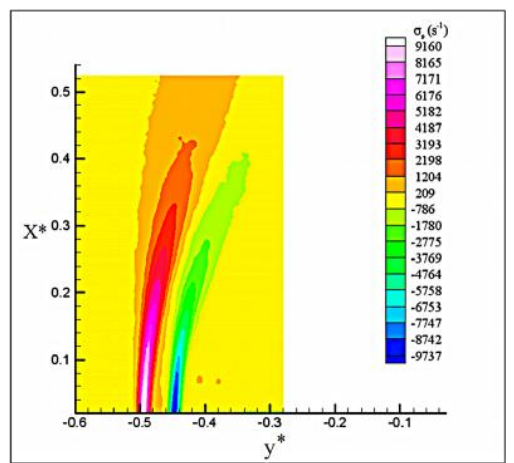

c)

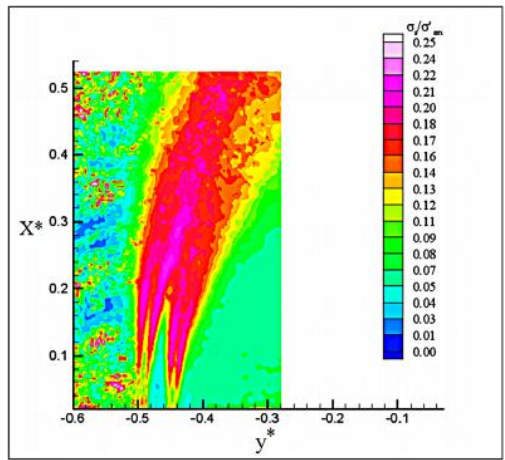

$\left.c^{\prime}\right)$

Fig.8 Mean vorticity field a) and rms vorticity a'), mean stress b) and rms stress b'), mean shear c) and rms shear c') for $U_{0}=8 \mathrm{~m} / \mathrm{s}$.

The two mixing layers are well described from mean vorticity field and the internal mixing layer is more curved than the external one. Moreover, the two mixing layers are getting further each other. Vorticity values are identical in the two mixing layers. The vorticity rms shows that initial fluctuations of vorticity are greater into the internal mixing layer but when, $0.2<x^{*}<0.4$, they become greater in the external one. Mean strain stress is greater in the external mixing layer for $0.1<x^{*}<0.4$. In the internal mixing layer, the fluid is strained from the output of the flow. A high strain stress value is also obtained between the obstacle and the recirculation zone. This strain is relatively constant because rms values are around $20 \%$ whereas they are $80 \%$ to $100 \%$ in the external mixing layer. Mean shear 
stress is greater at the exit of the flow, what is predictable. The shear between the flow and the surrounding air is more stable in the external mixing layer.

All these differences between external and internal shear layer are caused by the presence of the $D_{i}$ obstacle.

The Rayleigh criterion accounts for the influence of the curvature on the stability of a mixing layer (Saric 1994). The Rayleigh criterion $R_{a}$ is defined by $R_{a}=\frac{\partial G^{2}}{\partial y}$, where $G$ is the circulation of fluid particle.

When $R_{a}$ is negative, the mixing layer is unstable. In our configuration, this criterion is negative in the external mixing layer while $R_{a}$ is positive in the internal mixing layer, according to the velocity profile and the shear layers curvature in both cases (Figure 9). This can explain that rms velocities are greater in the external mixing layer (Figure 9(b)).

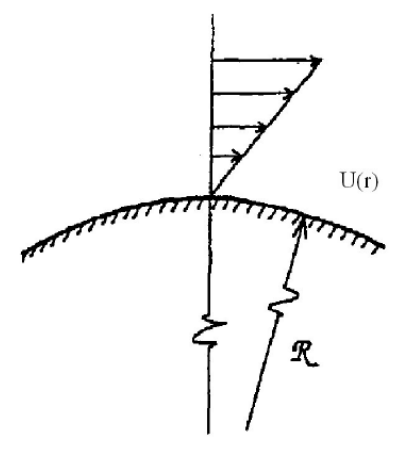

(a) stable

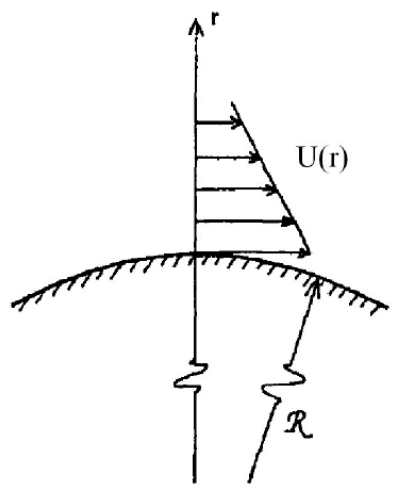

(b) unstable

Fig.9 Influence of the curvature on the stabilization of a mixing layer: (a) Inner shear layer, (b) External shear layer (Saric (1994)).

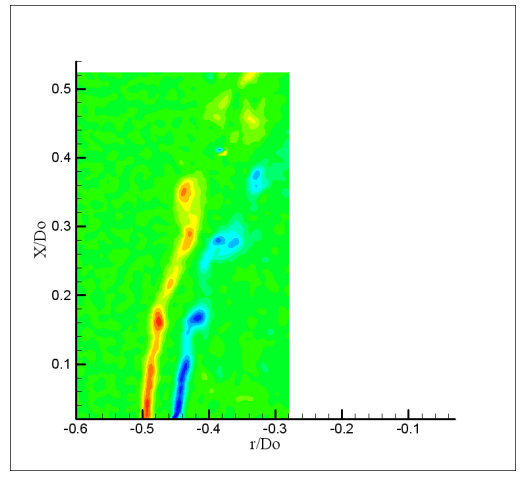

(a)

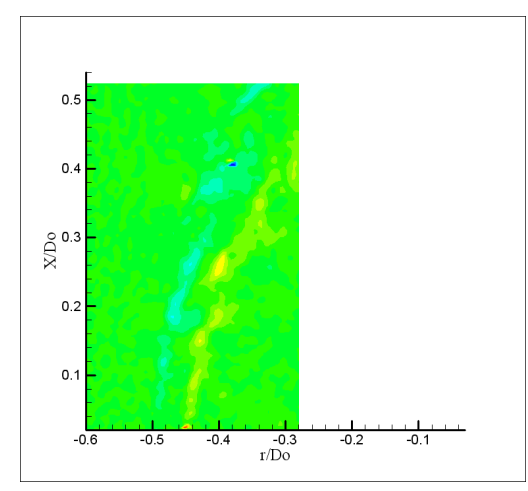

(b)

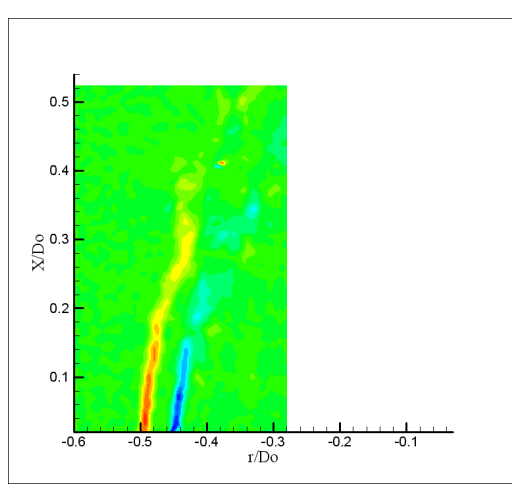

(c)

Fig.10 Examples of (a) vorticity fields, (b) strain fields and (c) shear fields for $U_{0}=8 \mathrm{~m} . \mathrm{s}^{-1}$. 
Samples of instantaneous vorticity, strain and shear fields are plotted on figure 10 for $U_{0}=8 \mathrm{~m}_{\mathrm{s}}^{-1}$. For $x^{*}<0.1$ to 0.18 , shear stress is high between the flow and the surrounding air, or between the flow and the recirculation zone. Downstream, Kelvin-Helmholtz vortices are created in the two shear layers. Temporal creation of vortices is not synchronized into the two shear layers. Contrary to a round jet flow, the two vortices, in the axisymetric plane of the flow, are not created on the same shear layer. Kelvin-Helmholtz vortices are characterized by a Strouhal number $S t_{\theta}$ calculated from momentum boundary layer thickness $\theta$, width of the lip $e$ and frequency $f$. The momentum boundary layer of the external shear layer $\theta_{e}$ and the internal shear layer $\theta_{i}$, respectively the momentum boundary layer of the external and internal shear layer, are different because the initial conditions of the shear are not the same (figure 10). Into each vortex street, the distance between each vortex is not a constant. Pairing processes also occur in the two mixing layers.

The temporal study of the two mixing layers have been realized with a single probe hot-wire anemometry. Measurements have been performed in the two mixing layers from $x^{*}=0.025$ to $x^{*}=0.5$ for $U_{0}$ equal to 8,15 and $30 \mathrm{~m} \cdot \mathrm{s}^{-1}$. Longitudinal velocity Power Spectral Densities (PSD) are plotted on figure 11 for $U_{0}=15 \mathrm{~m} \cdot \mathrm{s}^{-1}$. PSD have been computed from velocity fluctuations measured on the axis of the external and internal mixing layer and on the axis of the annular potential core flow. PSD are different in the initial internal and external mixing layers. Figure 11 presents results for the PSD analysis of the inner mixing layer, because high frequencies are more energetic in the case of the internal layer. Kelvin-Helmholtz instability frequency appears and is equal to $1210 \mathrm{~Hz}$. For $x^{*}=0.2$, it appears a weak "hill" for frequency around $610 \mathrm{~Hz}$ and the peak at $1210 \mathrm{~Hz}$ starts to disappear. This is characteristic of pairing process. By calculating the Strouhal number characterized with both the momentum boundary layer $\theta$ and the width $e$, it can be seen that $S t_{\theta}$ and $S t_{e}$ are independent of $U_{0}$ and are respectively equal to 0.012 and 0.23 in the two shear layer. These numbers are characteristic of Kelvin-Helmholtz instabilities. The Strouhal number value $S t_{\theta}$ is similar to the analytical value 0.016 obtained by Michalke (1982), and is in 


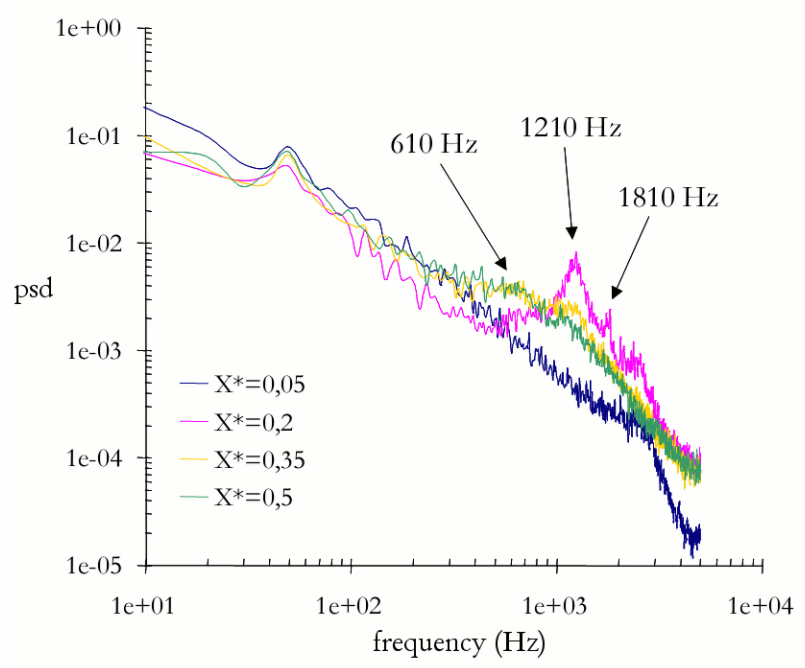

accordance with Hussain and Zaman (1980) round jet experimental measurements.

Fig.11 Power Spectral Density of longitudinal velocity for $U_{0}=15 \mathrm{~m} \cdot \mathrm{s}^{-1}$ measured at different distances $x^{*}$ from the nozzle outet, in the inner mixing layer.

Calculations have lead to the determination of convection velocity of primary vortices in the two mixing layers of the initial zone of the annular jet flow. The method is based on the ratio between a distance and a characteristic time if the hypothesis that the velocity of the vortex is a constant. Each vortex of each mixing layer has been spatially localized by using Graftieux criterion (Graftieux et al. 2000). The $Q$ coefficient fields have not been used to localize the vortices because $Q$ value doesn't depend on rotation direction of vortices, contrary to Graftieux criterion. A Power Density Function (PDF) of inter distances between two consecutive vortices can be computed from the statistic of P.I.V. fields and is plotted on figure 12. In the external mixing layer, the mean inter-distance is equal to $0.06 D_{o}$ for each exit velocity $U_{0}$. In the internal mixing layer, the maximal value of the PDF depends on the output velocity of the flow. The characteristic time of the convection of the vortices are obtained from Kelvin-Helmholtz instability frequency. Convection velocities $U_{c}$ are thus calculated and the nondimensional convection velocity $U_{c}^{*}=\frac{U_{c}}{U_{0}}$ is thus equal in the external mixing layer to $0.34,0.28$ and 0.28 to respectively $U_{c}$ equal to 8,15 and $30 \mathrm{~m} / \mathrm{s}$. In their internal mixing layer, $U_{c}^{*}$ is equal to $0.40,0.26$ and 0.23 . The external shear layer is characterized by the initial flo interacting with the surrounding air at rest. The internal mixing layer is characterized by the initial jet flow interacting with the 
rotating flow of the recirculation zone. The velocity gradient is then higher in the external mixing layer than in the internal mixing layer. Thereby, the convection velocity of Kelvin-Helmholtz vortices is then higher in the internal mixing layer. It also appears that $U_{c}$ decreases with $U_{0}$.
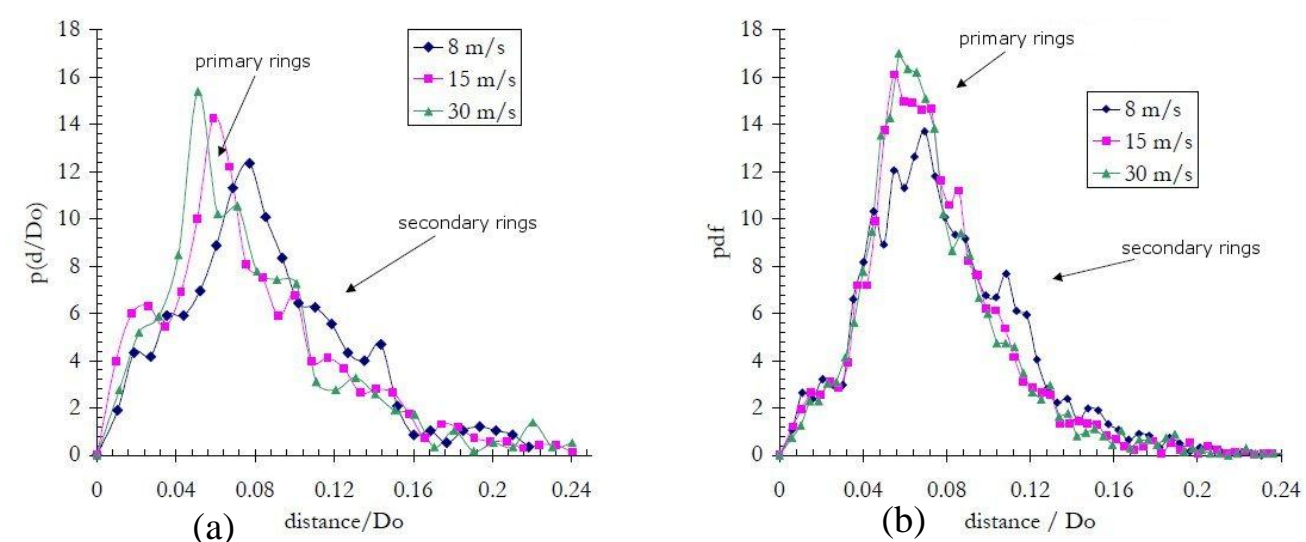

Fig.12 Probability density function of vortices inter-distance in a) the internal mixing layer, b) the external mixing layer.

\section{Study of the secondary instabilities in mixing layers}

Tomographic images of a transversal section of the flow, at different distances from the outlet of the nozzle (figure 13) have been recorded. These transversal cuts show the instabilities development and the vortices evolution for different outlet velocities of the flow from $8 \mathrm{~m} \cdot \mathrm{s}^{-1}$ to $30 \mathrm{~m} \cdot \mathrm{s}^{1}$ (from $\operatorname{Re}_{e}=1368$ to $\operatorname{Re}_{e}=5130$ ), in the recirculation zone. At the distance $x^{*}=0.25$ from the outlet of the nozzle, images are recorded in the middle of the recirculation zone (figure 14). This position shows the apparition of primary and secondary structures which are more developed for $x^{*}=0.375$, in the third quarter of the recirculation zone. Kelvin-Helmholtz vortices are clearly highlighted and contra-rotative vortices pairs created by secondary instabilities pull away from the round jet core. When the Reynolds number increases, these primary and secondary structures are more numerous and smaller. Kelvin-Helmholtz vortices are then less identifiable, secondary structures seem to take a bigger part in the flow development. Figure 15 also represents transversal cuts of the flow from the end of the recirculation zone, from the stagnation point plane to the fully developed zone for each studied velocity. Downstream the stagnation point position, $x^{*}=0.5$, structures are less 
identifiable and the flow is less tightened around the jet axis. In the transition zone, $x^{*}=0.75$, jets with different outlet velocities can no longer be distinguished. Secondary structures in a round jet flow plays a significant role in mechanisms used in ventilation. It is then interesting to study these structures more particularly.

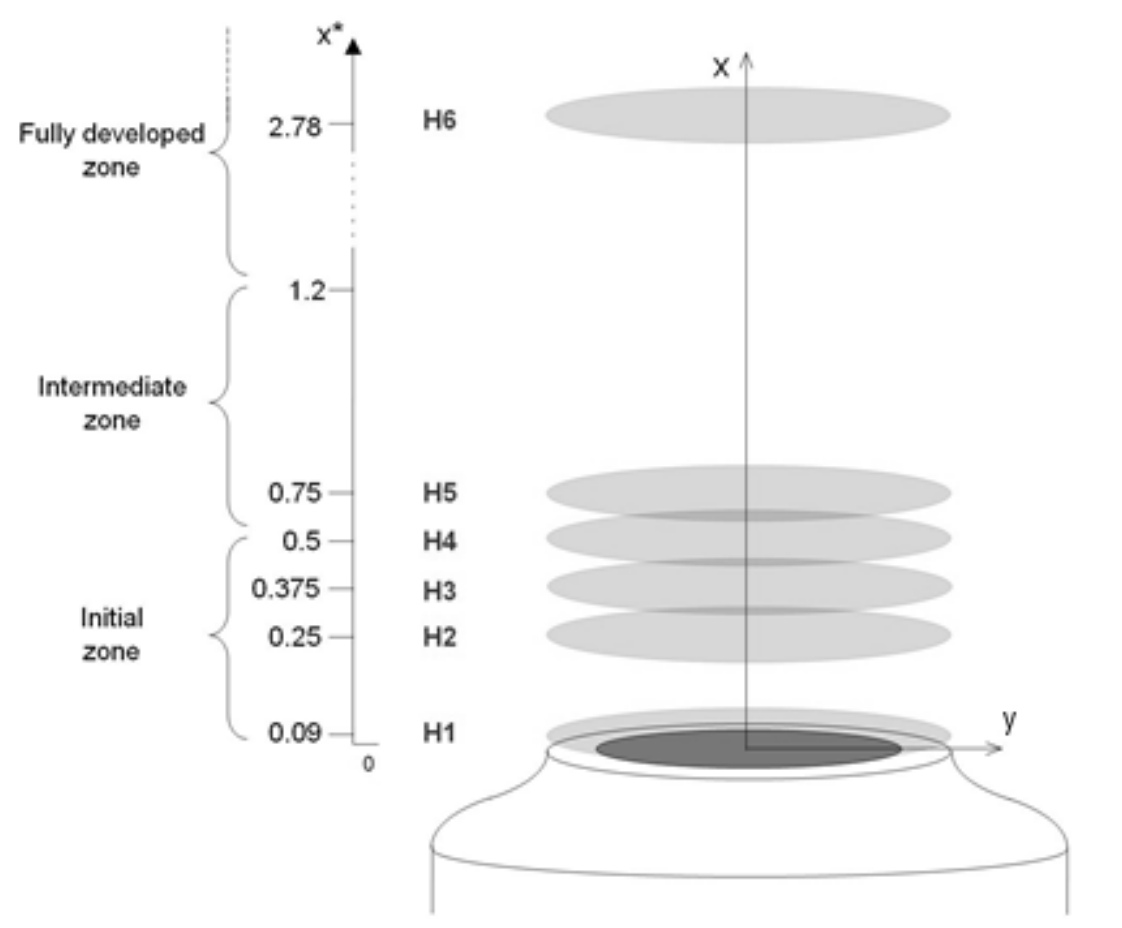

Fig.13 Diagram of transversal planes studied by fast tomography 


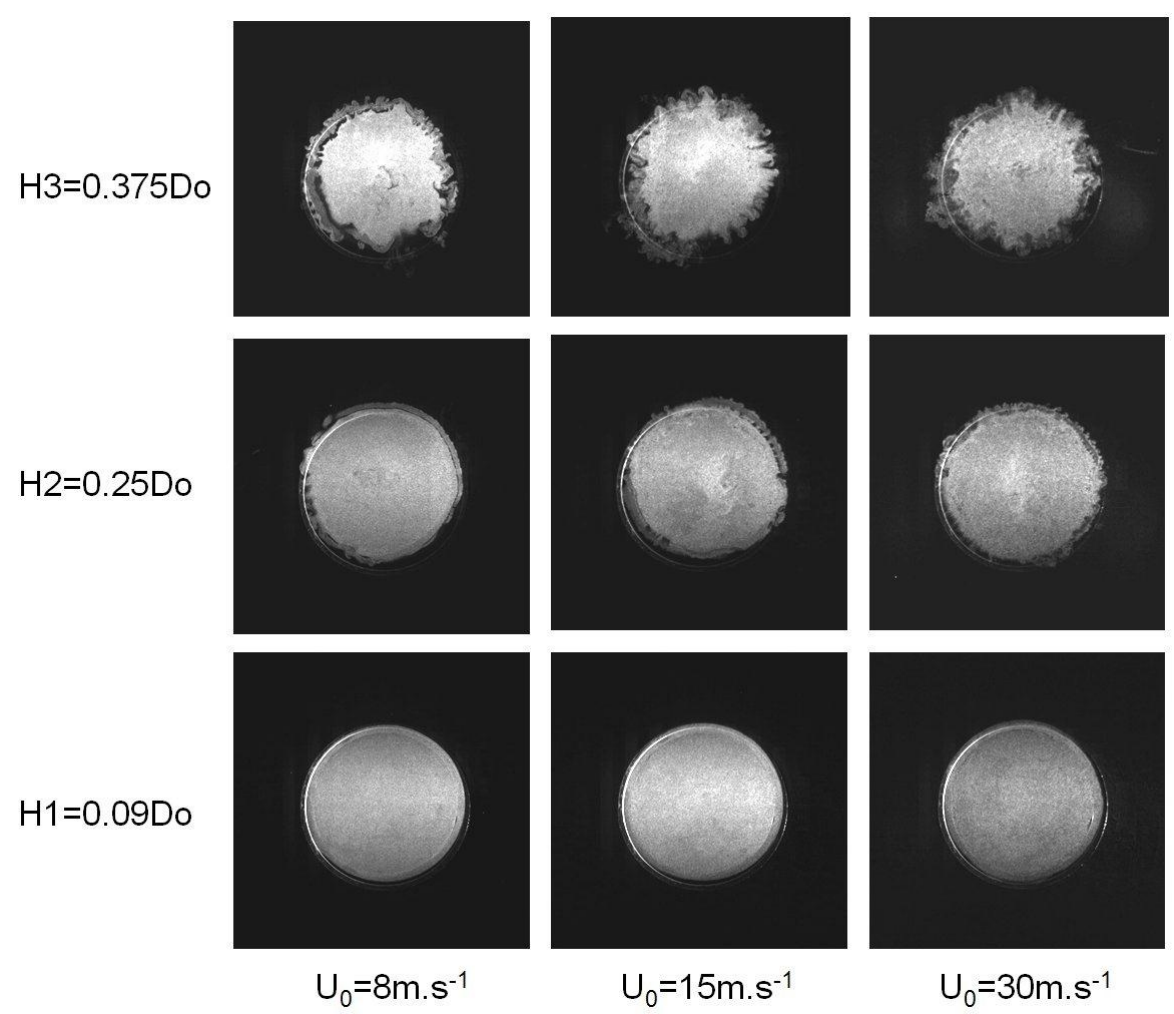

Fig.14 Transversal images of the annular jet flow in the recirculation zone, for different outlet velocities $U_{0}$

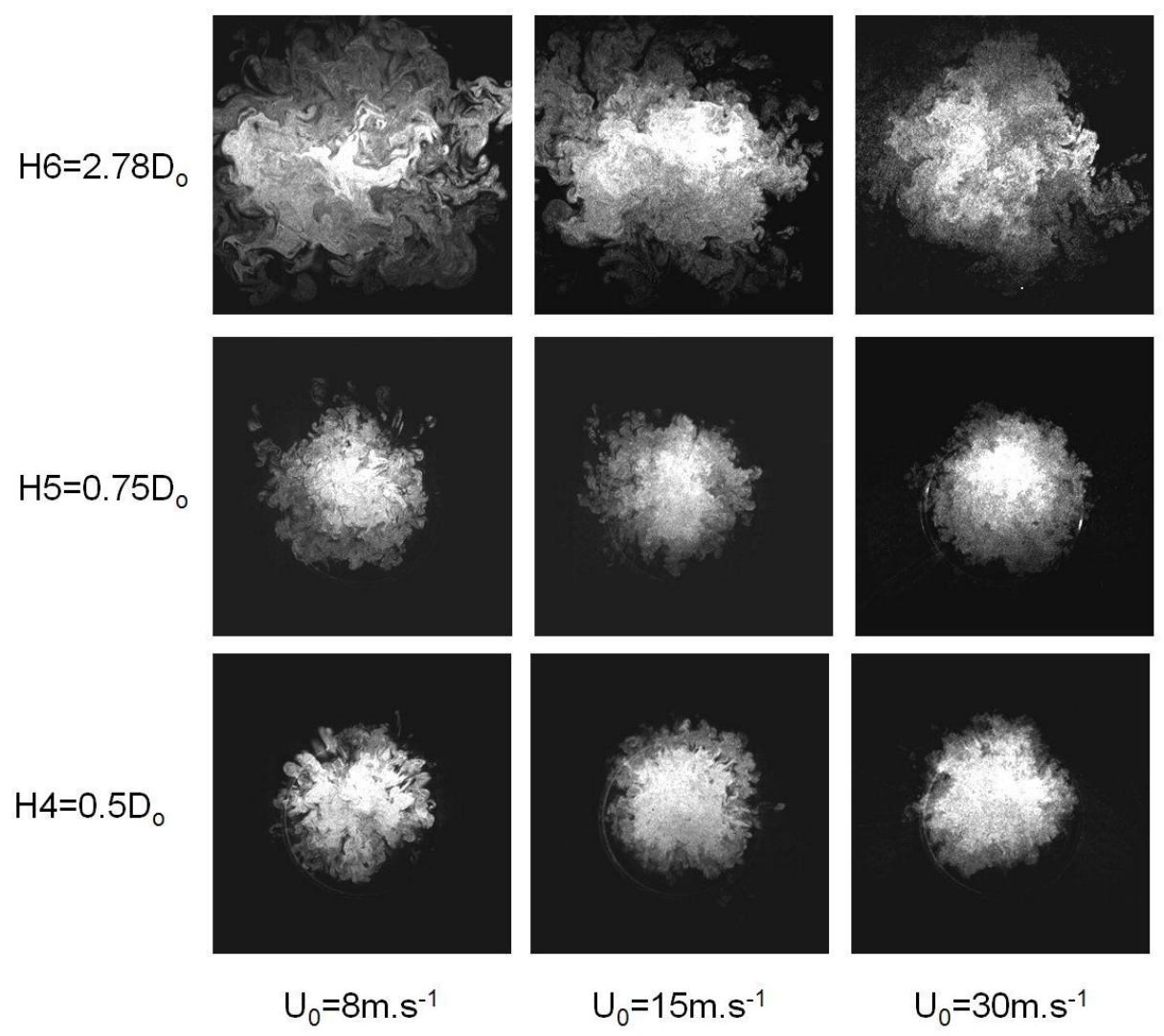

Fig.15 Transversal images of the annular jet flow from the stagnation plane to the fully developed zone, for different outlet velocities $U_{0}$ 
A Proper Orthogonal Decomposition (P.O.D.) analysis has been used on a thousand tomographic images of transversal cuts of the annular jet in order to highlight coherent structures developed in different slices of the flow. Results of a direct method calculation of P.O.D. are presented on figure 16 to study the middle of the recirculation zone $\mathrm{H}_{2}\left(x^{*}=0.25\right)$, where instabilities begin to create structures. Secondary structures are then localized on the external ring of the jet but we can see this ring on each mode. This means that the jet periphery consists in structures with different energetic weights. Downstream the initial zone, in the transition zone (figure 17) and in the fully developed zone (figure 18), the flow seems to be more disordered than in the previous part of the jet but in contrary to the case of the initial zone, in the transition and in the fully developed zones, structures can be decomposed on different modes and look like organized in preferred axes. This observation is essential in the study of the annular jet development. As a matter of fact when we want to study a passive control of the flow by modifying the central obstacle geometry by example, organization of the flow downstream the initial zone and the preferred axes could be different. Mode 0 corresponds to the mean flow image but modes 1 and 2 correspond to the largest coherent structures of the flow. When we observe these modes and their evolution according the distance from the nozzle outlet $\mathrm{H}^{*}$, we can detect some preferred axes, around which these structures develop preferably. Figures 17 and 18 show that these structures develop in two or three zones of the flow, according to the distance from the nozzle outlet, for the largest structures (mode 1). The detection of these transversal preferred axes could also lead to the hypothesis of an average asymmetry of the annular jet flow. 

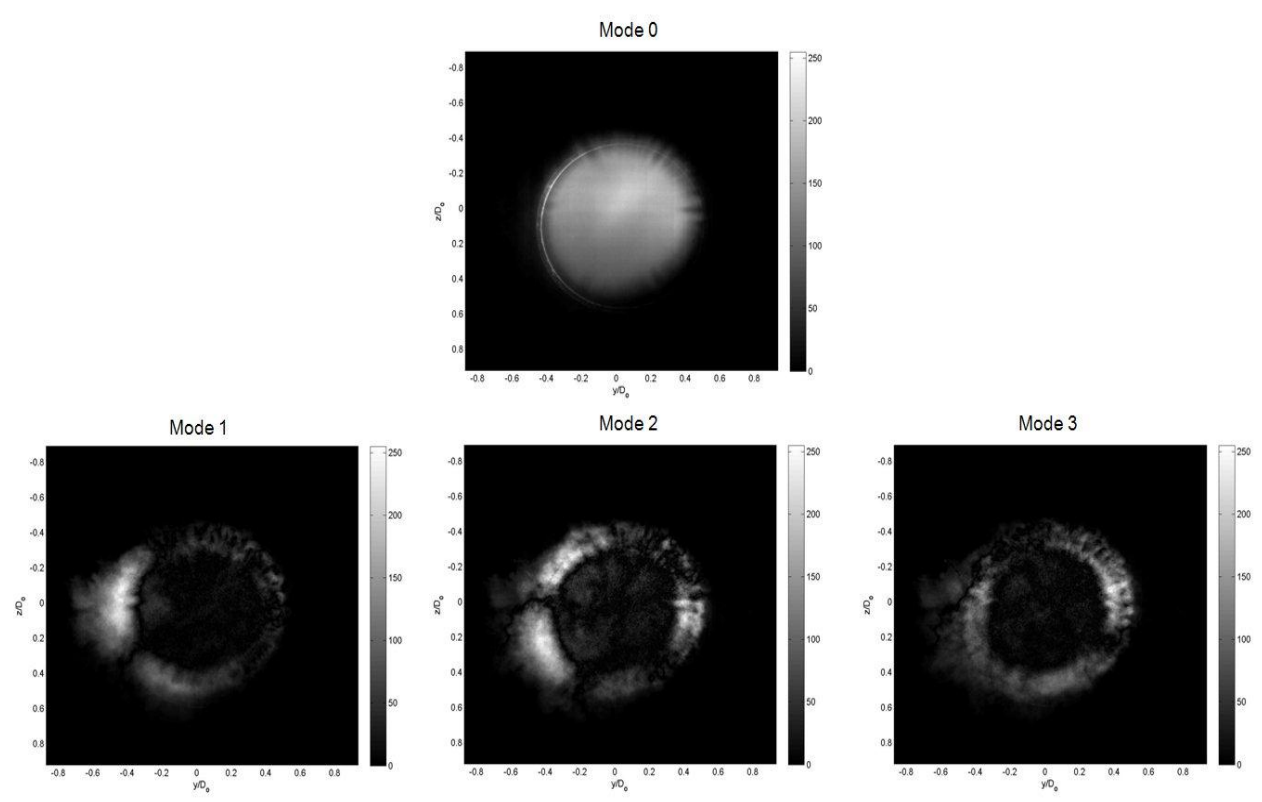

Fig.16 Proper modes of an annular jet with $U_{0}=15 \mathrm{~m} \cdot \mathrm{s}^{-1}$ calculated on 1000 images recorded at the distance $H_{2}=0.25 D_{o}$ from the nozzle outlet

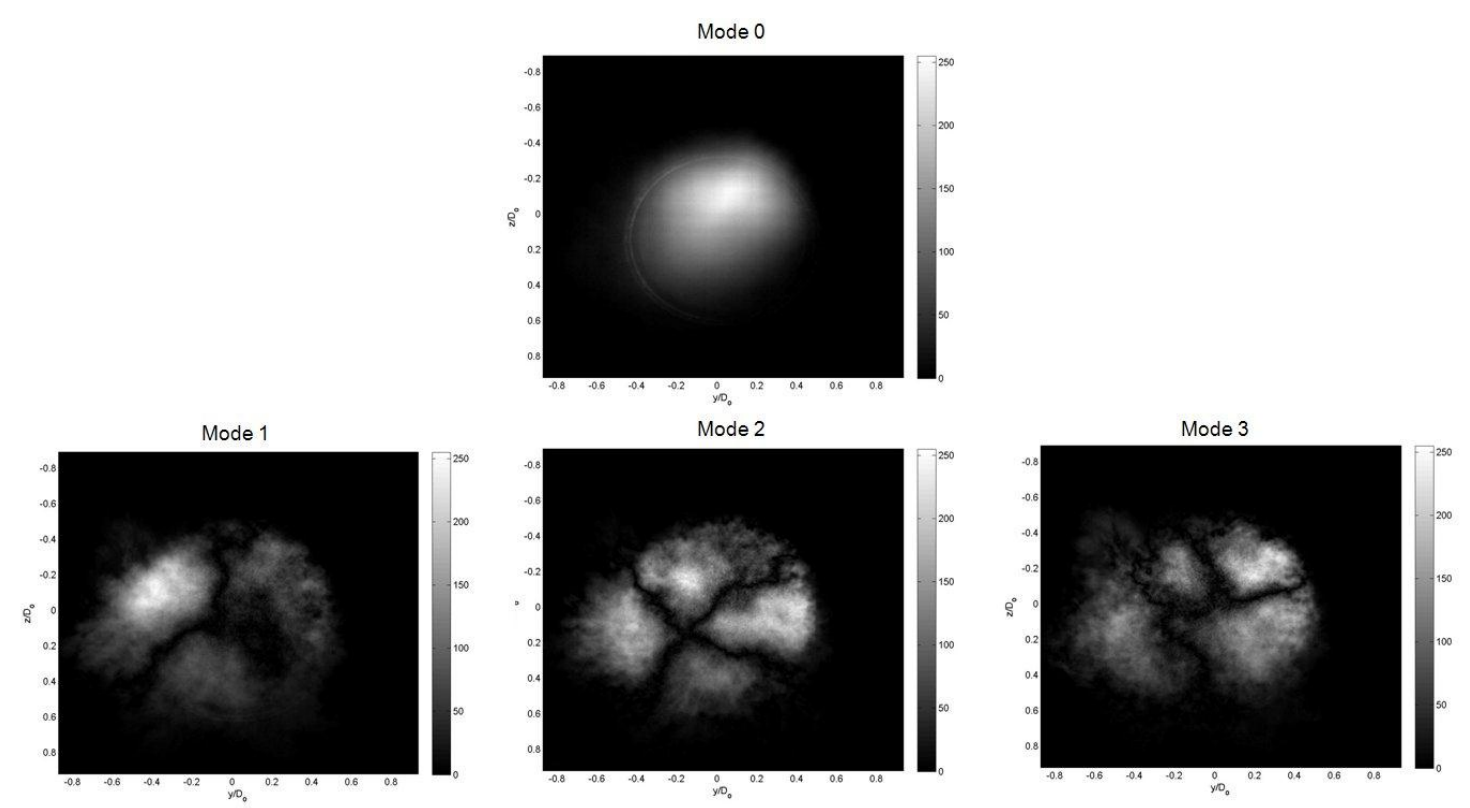

Fig.17 Proper modes of an annular jet with $U_{0}=15 \mathrm{~m} \cdot \mathrm{s}^{1}$ calculated on 1000 images recorded at the distance $H_{5}=0.75 D_{o}$ from the nozzle outlet 

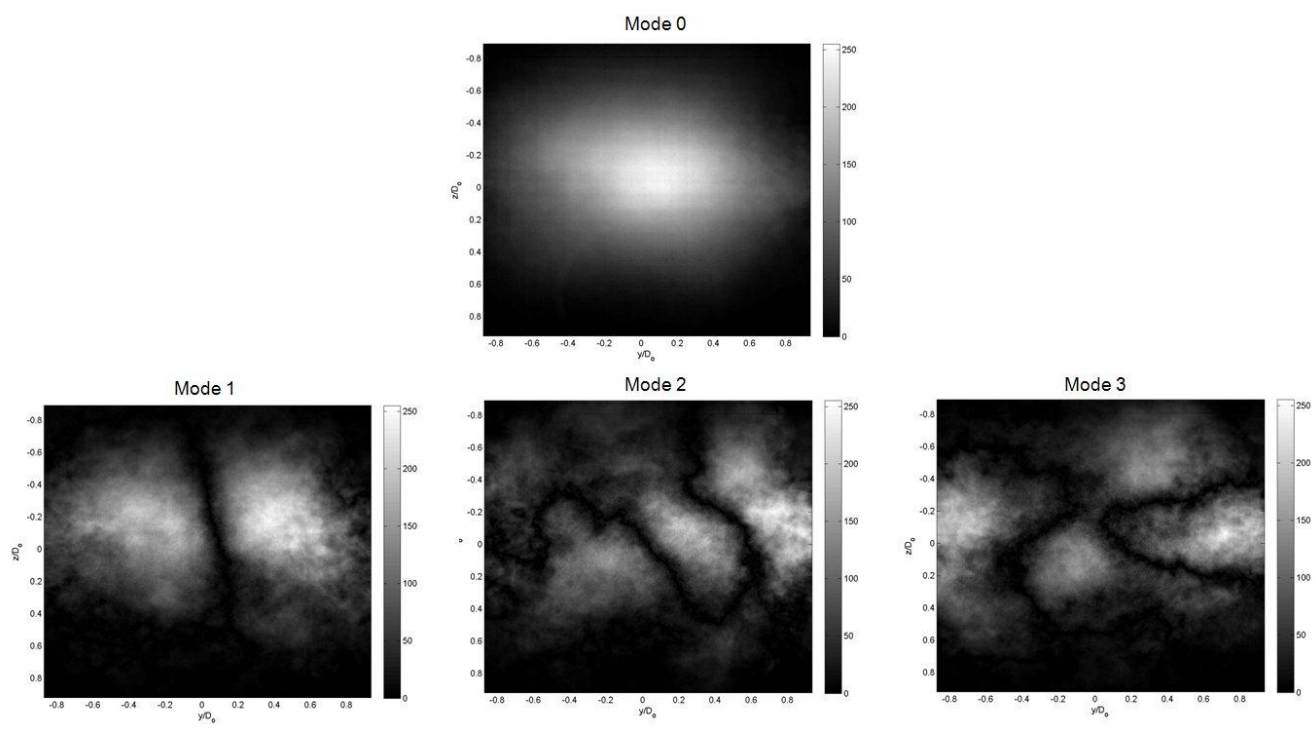

Fig.18 Proper modes of an annular jet with $U_{0}=15 \mathrm{~m} \cdot \mathrm{s}^{-1}$ calculated on 1000 images recorded at the distance $H_{6}=2.78 D_{o}$ from the nozzle outlet

\section{Average asymmetry.}

Each studied velocity profile represents a diameter of a flow transversal plane. Results of velocity measurements are shown on figure 19 in the middle $\left(H_{2}\right)$ and at the end $\left(\mathrm{H}_{4}\right)$ of the recirculation zone. We can see then an asymmetry of the mean flow as it is underlined with the study of the tangential component of velocity for each azimuth angle shown on figure 20. Contrary to the hypothesis deduced from bi-dimensional velocity measurements in a longitudinal plane of the flow, here the jet seems to have preferred axes to oscillate. This remark completes observations of P.O.D. calculations on transversal tomographic images.

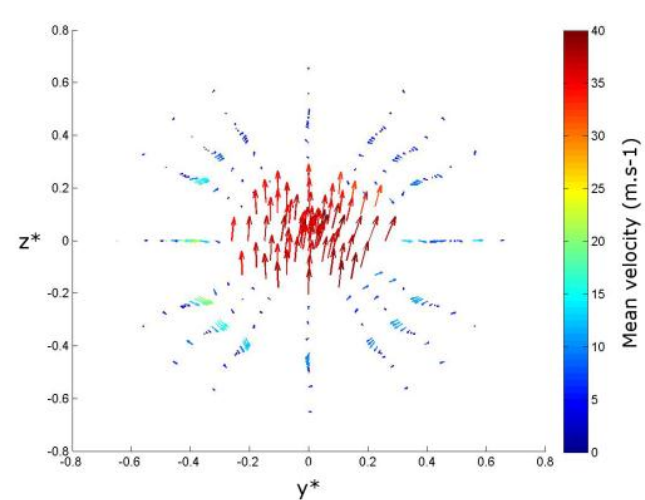

(a)

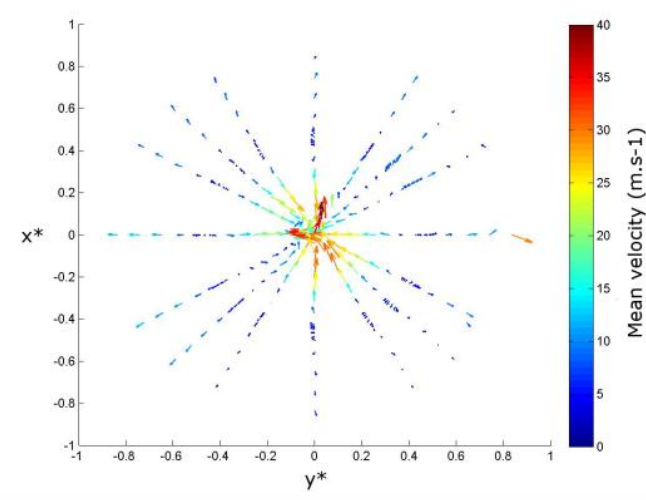

(b) 
Fig.19 Velocity fields measured by 3D LDA in slices of the flow, (a) in the middle of the recirculation zone $H_{2}\left(x^{*}=0.25\right)$ and $(\mathrm{b})$ at the end of the recirculation zone $H_{4}\left(x^{*}=0.5\right)$ of an annular jet with $U_{0}=30 \mathrm{~m} \cdot \mathrm{s}^{-1}$

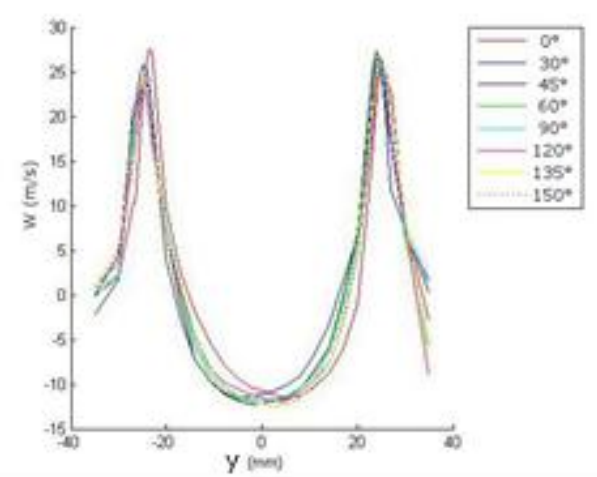

(a)

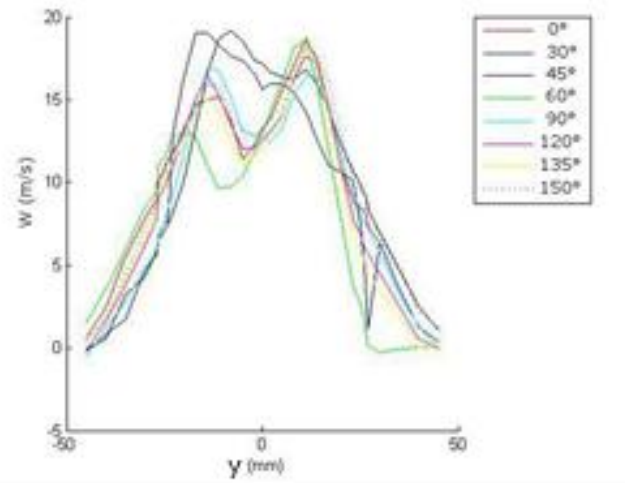

(b)

Fig.20 Tangential component $\mathrm{W}$ of average velocity measured with three-dimensional LDA for different azimuth angles (a) in $H_{2}$ and (b) in $H_{4}$ for $U_{0}=30 \mathrm{~m} \cdot \mathrm{s}^{-1}$

Del Taglia (2003) has already detect this loss of mean axisymetry with Reynolds Average Navier Stokes simulations (RANS) validated by three-dimensional L.D.A. measurements of an annular jet with great diameter ratios $(r=0.94)$. The author suggests that this average asymmetry could appear, if inertial forces are higher than pressure forces in the stagnation point, at the end of the recirculation zone, and if the stagnation point moves then with a distance higher than the jet thickness at the outlet of the nozzle. This phenomenon could appear in particular configurations characterized by a parameter $\gamma$ defined as:

$$
\gamma=\left(1-\frac{1}{\operatorname{Re}_{D_{o}}\left(\frac{D_{o}^{2}}{D_{i}^{2}}-1\right)}\right)\left(\frac{1}{\frac{D_{o}}{D_{i}}-1}\right)
$$

where $\operatorname{Re}_{D o}$ is the Reynolds number based on the external diameter $D_{o}$. If $3.18 \leq \gamma \leq 16$, then the flow will presents an average asymmetry in the recirculation zone. Within our configuration, with a diameter ratio $r=0.91$ and our range of Reynolds numbers $\left(28000<\operatorname{Re}_{D_{o}}<108000\right), \gamma$ is equal to 9.50. Our experimental device is a candidate to the loss of average symmetry. Figure 21 makes comparison between the mean asymmetry observed with our large diameter ratio configuration (Figure 21(a)) and the mean asymmetry observed by 
Del Taglia et al. (2004) with a Reynolds Stress Model of Speziale Sarker and Gatski simulation (Figure 21 (b)).

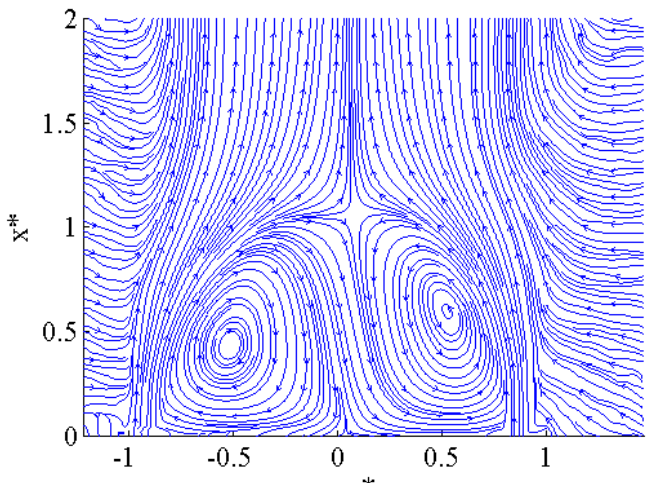

(a)

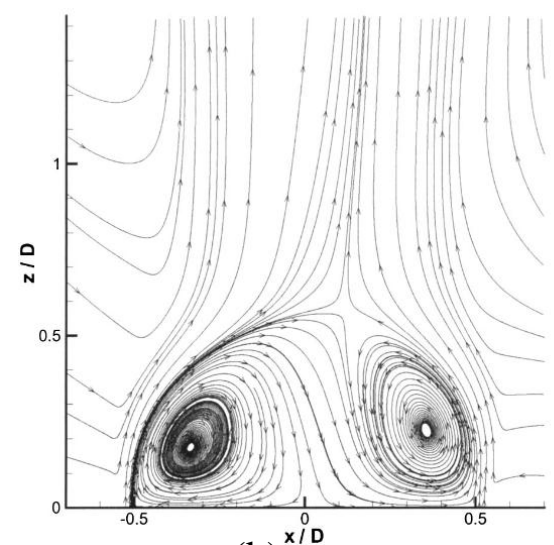

(b)

Fig.21 Streamlines of the average flow of an annular jet with large diameter ratio (a) obtained with 2D P.I.V. measurements on our configuration $(r=0.91$ and $\operatorname{Re}_{D_{o}}=54000$ ) and (b) obtained by SSG-RSM simulation by Del Taglia et al. (2004) $\left(r=0.94\right.$ and $\left.\operatorname{Re}_{D_{o}}=72000\right)$.

At the end of the recirculation zone, in the plane of the stagnation point, the flow presents some preferred axes. This selection of preferred direction is observed in the study of coherent structures too. The asymmetry of the flow seems to be a result of the development of secondary structures on the rims of the jet. The annular jet behavior could be then governed by mixing layer instabilities. In order to set up a passive control of the annular jet flow it would be interesting to act on mixing layer instabilities rather than wake instabilities.

\section{Conclusion}

An annular jet with a large diameter ratio has been studied in order to meet manufacturer needs. As a matter of fact, this flow could be a severe handicap in many industrial processes. For example, annular jets are used in glass fibers process. For this particular industrial application, the process velocity must be high but the fibers diameter must be constant too, avoiding the fibers breakage. Waste glass are several millions tons in France. These manufacturing defects originate mostly from aerodynamic forces and flow instabilities. In order to prevent this problem, annular jet flow has to be perfectly understood, so has to 
provide a good solution, easy to set up. First the initial zone of an annular jet with a large diameter ratio (for glass fibers process, $r>0.95$ ) has been investigated, from the nozzle outlet to the end of the recirculation zone located downstream the central obstacle. Then, the study of the mixing layer and its instabilities has opened the way to a new explanation of the flow average asymmetry in particular experimental configurations. This asymmetry depends on initial velocity of the jet at the nozzle outlet and diameter ratio as explained by Del Taglia (2003). Experimental measurements and P.O.D. data analysis show that mixing layer instabilities could be predominant in comparison with wake instabilities. A way to control these flows could therefore be to modify the nozzle external zone. Doing so may act on mixing layer instabilities and suppress the flow average asymmetry. In fact, the annular jet flow can no more be studied with bi-dimensional measurements because these ones mask the secondary instabilities created in the braid of Kelvin-Helmholtz vortices, whereas these instabilities play a significant role in the flow development and more particularly in the global destabilization of the flow. In order to complete this study, PIV measurements in transversal planes of the flow could be performed. Indeed, the mean asymmetry of the flow is not completely understood and further measurements are needed. This study could also lead to a passive or active control of the flow, by modifying the nozzle geometry or by adding some actuators to act on Kelvin-Helmholtz development.

\section{References}

Aly MS and Rashed MII (1991) Experimental investigation of an annular jet. Journal of Wind Engineering and Industrial Aerodynamics 37:155-166

Benney D (1961) A non-linear theory for oscillations in a parallel flows. Journal of Fluid Mechanics 10:209-236

Bernal L and Roshko A (1986) Streamwise vortex structure in plane mixing layers. Journal of Fluid Mechanics 170:499-525

Brendel M (2002) CFD analysis of laboratory exhaust fans and applications. Ashrae winter annual meeting

Brown G and Roshko A (1974) On density effects and large structures in turbulent mixing layers. Journal of Fluid Mechanics 64:775-814 
Chigier NA and Beer JM (1964) The flow region near the nozzle in double concentric jets. Journal of Basic Engineering, December:797-804

Crow SC and Champagne FH (1971) Orderly structure in jet turbulence. Journal of Fluid Mechanics 48 :547-591

Danlos A, Rouland E and Patte-Rouland B (2008) Proper Orthogonal Decomposition used for aerodynamic study and active control of annular jet instabilities using acoustic excitations. $6^{\text {th }}$ IASME/WSEAS International Conference on Fluid Mechanics and Aeroydnamics, Rhodes, Greece

Davies TW and Beer JM (1971) Flow in the wake of bluff-body flame stabilizers. Thirteenth symposium on combustion, the Combustion Institute 631-638

Del Taglia C (2003) Numerical investigation of the non-reacting unsteady flow behind a disk stabilized burner with large blockage. $\mathrm{PhD}$ from the university of Rome "La Spienza"

Del Taglia C, Blum L, Ventikos Y, Poulikakos D (2004) Numerical and experimental investigation of an annular jet flow with large blockage. Journal of Fluids Engineering 126:375384

Foss JF, Prevost RJ, Bade KM and Levasseur A (2003) The velocity field of an annular jet with cross-member. Proceeding of FEDSM'03 Honolulu

Gaster M and Bradbury LJS (1976) The measurement of the spectra of highly turbulent flows by a randomly triggered pulsed-wire anemometer. Journal of Fluid Mechanics 77:499-509

Godard G, Rouland B, Paranthoen P and Lecordier JC (1998) Développement d'un jet annulaire continu ou partiellement obturé. 6eme congrès Francophone de Vélocimétrie Laser, Saint Louis

Graftieux L ,Michard M and Grosjean N (2000) Combining PIV, POD and vortex identification algorithms for the study of unsteady turbulent swirling flows. EuroMech 411, Europeen Mechanics Society

Holmes P, Lumley JL, Berkooz G (1996) Turbulence, Coherent Structures, Dynamical Systems and Symmetry. Cambridge Monographs on Mechanics

Huang RF, Chen CF, Lin CL and Bear GM (1994) Smoke wire flow visualization of the nearwake region behind a circular disk at low Reynolds numbers. Experiments in Fluids, vol. 17, pp. 259-266. 
Hussain AKMF and Ramjee V (1976) Effects of the axisymmetric contraction shape on incompressible turbulent flow. Transactions of the ASME 58-69

Hussain AKMF and Zaman KBMQ (1980) Vortex pairing in a circular jet under controlled excitation. Part 2. Coherent structure dynamics. Journal of Fluid Mechanics 101/3:493-544

Ko NWK and Chan WT (1978) Similarity in the initial region of annular jets :three configurations. Journal of Fluid Mechanics 84/ 4: 641-656

Ko NWM, Lau KK and Lam KM (1998) Dynamics of interaction modes in excited annular jets. Experimental Thermal and Fluid Science 1:319-338

Ko NWK and Leung KC (1982) Covariance measurements in the initial region of an annular jet. Journal of Sound and Vibration 80 (3):339-354

Lalizel G (2004) Caractérisation expérimentale de l'aérodynamique d'un jet annulaire à très grand rapport de diamètres. Phd thesis, University of Rouen, France.

Li X and Tankin R (1987) A study of cold and combusting flow around bluff-body combustors. Combustion science and technology 52:173-206

Michalke A and Hermann G (1982) On the inviscid instability of a circular jet with external flow. Journal of Fluid Mechanics 114:343-359

Patte-Rouland B, Lalizel G, Moreau J and Rouland E (2001) Flow analysis of an annular jet by Particle Image Velocimetry and Proper Orthogonal Decomposition. Measurement Science and Technology 12:1404-1412

Pierrehumbert R and Widnall S (1982) The two and three-dimensional instabilities of a spatially periodic shear layer. Journal of Fluid Mechanics 245:642-668

Saric WS (1994) Görtler Vortices. Ann Rev Fluid Mech 26:379-109

Sheffer RW, Namazian M, Kelly M and Perrin M (1996) Effect of confinement on Bluff-Body burner recirculation zone characteristics and flame stability. Combustion Science and Technology 120:185-211

Vanierschot M, Van Den Bulck E (2011) Experimental study of low precessing frequencies in the wake of a turbulent annular jet, Experiments in Fluids, 50:189-200. 
Van Maanen, HRE, Nobach, H, Benedict LH (1999) Improved estimator for the slotted autocorrelation function of randomly sampled LDA data. Measurement Science and Technology 10 no. 1: L4-L7

Warda HA, Kassab SZ, Elshorbagy KA and Elsaadawy EA (1998) An experimental investigation of the near field region of free turbulent round central and annular jets. Flow measurement and Instrumentation 10:1-14

Weiss F (1999) Etude expérimentale de la diffusion de la chaleur en aval d'une source linéaire place dans une allée de Bénard-Von Karman. Thèse de doctorat de l'Université de Rouen 